\title{
Continuous Time Mixed State Branching Processes and Stochastic Equations 1
}

\author{
Shukai Chen and Zenghu Li \\ Laboratory of Mathematics and Complex Systems, \\ School of Mathematical Sciences, Beijing Normal University, \\ Beijing 100875, People's Republic of China \\ E-mails: skchen@mail.bnu.edu.cn and lizh@bnu.edu.cn
}

\begin{abstract}
A continuous time mixed state branching process is constructed as the scaling limits of two-type Galton-Watson processes. The process can also be obtained by the pathwise unique solution to a stochastic equation system. From the stochastic equation system we derive the distribution of local jumps and the exponential ergodicity in Wasserstein-type distances of the transition semigroup is given. Meanwhile, we study immigration structures associated with the process and prove the existence of the stationary distribution of the process with immigration.
\end{abstract}

Key words and phrases. Mixed state branching process; weak convergence; stochastic equation system; Wasserstein-type distance; stationary distribution.

\section{Introduction}

Branching processes were introduced as probabilistic models describing the evolution of populations. The study of branching processes were first initiated by Bienaymé (1845), Galton and Watson (1874), independently, which was known as discrete time and discrete state GaltonWatson processes (GW-processes). To be more realistic, several naturally generalized processes, continuous time discrete state branching processes (DB-processes) with or without immigration and continuous time continuous state branching process (CB-processes) with or without immigration were introduced and studied by researchers.

The DB-processes are continuous time discrete state Markov processes with lifetimes are independent and exponentially distributed random variables. There have been abundant works on the understanding of DB-processes including the construction, properties of moments, limit theorems and so on, we refer to [1] for the details. The approach of stochastic equations for branching processes has been developed in the past decades. Let $\mathbb{N}=\{0,1,2, \ldots\}$ and $\sharp(\cdot)=\sum_{i} \delta_{i}(\cdot)$ be a counting measure on $\mathbb{N}$. Let $X=\left\{X_{t}: t \geq 0\right\}$ be a DB-process with immigration with branching rate $c>0$, offspring distribution $\left(p_{i}: i \in \mathbb{N}\right)$, immigration rate $\eta>0$ and immigration distribution $\left(q_{i}: i \in \mathbb{N}\right)$. The two distributions satisfy $\sum_{k=1}^{\infty} k p_{k}<\infty$ and $\sum_{k=1}^{\infty} k q_{k}<\infty$. It is known that $X$ can be obtained as a pathwise unique strong solution of the following stochastic equation:

$$
X_{t}=X_{0}+\int_{0}^{t} \int_{\mathbb{N}} \int_{0}^{X_{s-}}(z-1) M(\mathrm{~d} s, \mathrm{~d} z, \mathrm{~d} u)+\int_{0}^{t} \int_{\mathbb{N}} z N(\mathrm{~d} s, \mathrm{~d} z),
$$

\footnotetext{
${ }^{1}$ Supported by the National Key R\&D Program of China (No. 2020YFA0712900) and the National Natural Science Foundation of China (No.11531001).
} 
where $X_{0}$ is a random variable taking values in $\mathbb{N}, M(\mathrm{~d} s, \mathrm{~d} z, \mathrm{~d} u)$ is a Poisson random measure on $(0, \infty) \times \mathbb{N} \times(0, \infty)$ with intensity measure $c p_{z} \mathrm{~d} s \sharp(\mathrm{d} z) \mathrm{d} u, N(\mathrm{~d} s, \mathrm{~d} z)$ is a Poisson random measure on $(0, \infty) \times \mathbb{N}$ with intensity mesure $\eta q_{z} \mathrm{~d} s \sharp(\mathrm{d} z)$, and $X_{0}, M(\mathrm{~d} s, \mathrm{~d} z, \mathrm{~d} u), N(\mathrm{~d} s, \mathrm{~d} z)$ are independent of each other. In particular, if $\eta \equiv 0, q_{z} \equiv 0$ for all $z \in \mathbb{N}$, this reduces to the DB-process. Moreover, here and in the sequel, we understand that for any $b \geq a \geq 0$

$$
\int_{a}^{b}=\int_{(a, b]}, \quad \int_{a}^{\infty}=\int_{(a, \infty)} .
$$

CB-processes were first introduced to applicably model the random evolution of large population dynamics in [16]. Denote the law on $\mathbb{D}([0, \infty),[0, \infty))$ by $\mathbb{P}_{x}$ for each initial value $x \geq 0$, the branching property of processes can be described by $\mathbb{P}_{x+y}=\mathbb{P}_{x} * \mathbb{P}_{y}$. The semigroup of CB-processes with immigration (CBI-processes) $\left(Q_{t}\right)_{t \geq 0}$ can be characterized uniquely by the Laplace transform:

$$
\int_{[0, \infty)} \mathrm{e}^{-\lambda y} Q_{t}(x, \mathrm{~d} y)=\exp \left\{-x v_{t}(\lambda)-\int_{0}^{t} \psi\left(v_{s}(\lambda)\right) \mathrm{d} s\right\}, \quad \lambda \geq 0, x \geq 0,
$$

where for any $\lambda \geq 0, t \mapsto v_{t}(\lambda)$ uniquely solves the following equation:

$$
v_{t}(\lambda)=\lambda-\int_{0}^{t} \phi\left(v_{s}(\lambda)\right) \mathrm{d} s, \quad t \geq 0
$$

the branching mechanism $\phi$ and immigration mechanism $\psi$ defined on $[0, \infty)$ take the form of

$$
\phi(z)=a z+\alpha z^{2}+\int_{0}^{\infty}\left(\mathrm{e}^{-z u}-1+z u\right) m(\mathrm{~d} u), \quad \psi(z)=b z+\int_{0}^{\infty}\left(1-\mathrm{e}^{-z u}\right) n(\mathrm{~d} u)
$$

with Lévy measures $m, n$ satisfying $\int_{0}^{\infty} u \wedge u^{2} m(\mathrm{~d} u)+\int_{0}^{\infty} u n(\mathrm{~d} u)<\infty$, and $a \in \mathbb{R}, b, \alpha \geq 0$. In particular, when $\psi \equiv 0$, this reduces to the CB-process. There are several ways to construct such processes. [11] proved that a diffusion process may arise in a limit theorem of GW-processes. In [19], the authors systematically studied the limit theorems of GW-processes with immigration and characterized the class of the limit process as a CBI-process, the conditions of the main theorem involved iterations of the probability generating functions. Some simpler conditions for the weak convergence were provided in [20], and [25] extended the result of [20] to a two-type CBI-process. Let $Y=\left\{Y_{t}: t \geq 0\right\}$ be a CBI-process, similarly, $Y$ can also be represented as a pathwise unique strong solution to the stochastic equation:

$$
\begin{aligned}
Y_{t}=Y_{0} & +\int_{0}^{t}\left(b-a Y_{s}\right) \mathrm{d} s+\sqrt{2 \alpha} \int_{0}^{t} \int_{0}^{Y_{s}} W(\mathrm{~d} s, \mathrm{~d} u)+\int_{0}^{t} \int_{0}^{\infty} z N(\mathrm{~d} s, \mathrm{~d} z) \\
& +\int_{0}^{t} \int_{0}^{\infty} \int_{0}^{Y_{s-}} z \tilde{M}(\mathrm{~d} s, \mathrm{~d} z, \mathrm{~d} u),
\end{aligned}
$$

where $Y_{0}$ is a random variable taking values in $\mathbb{R}_{+}, W(\mathrm{~d} s, \mathrm{~d} u)$ is a time-space white noise with intensity measure $\mathrm{d} s \mathrm{~d} u, M(\mathrm{~d} s, \mathrm{~d} z, \mathrm{~d} u)$ is a Poisson random measure on $(0, \infty)^{3}$ with intensity measure $\mathrm{d} s m(\mathrm{~d} z) \mathrm{d} u, N(\mathrm{~d} s, \mathrm{~d} z)$ is a Poisson random measure on $(0, \infty)^{2}$ with intensity measure $\mathrm{d} s n(\mathrm{~d} z), \tilde{M}(\mathrm{~d} s, \mathrm{~d} z, \mathrm{~d} u)=M(\mathrm{~d} s, \mathrm{~d} z, \mathrm{~d} u)-\mathrm{d} s m(\mathrm{~d} z) \mathrm{d} u$ is the compensated measure of $M(\mathrm{~d} s, \mathrm{~d} z, \mathrm{~d} u)$. Moreover, $Y_{0}, W, M$ and $N$ are independent of each other. We mention that the moment condition $\int_{0}^{\infty} z n(\mathrm{~d} z)<\infty$ was removed in [13]. The sample paths of $Y$ can also be obtained as a unique strong solution to a stochastic equation driven by Brownian motions and 
Poisson random measures, one can find the formulation (1.3) is more nice to analyse the flows of CBI-processes and other applications, see [6] for the specific construction. And we refer to [3, 5, 6, 13, 21, 23, 28, for the approach and further properties of the above stochastic equations. Based on stochastic equations established above, [14 studied the explicit expression of the distribution of jumps, some applications in Finance can be found in [17]. A two-type CBI-process obtained as a unique strong solution of a stochastic equation system was studied by [26, 27].

We can rewrite (1.3) without immigration part by extending the $M$ to a Poisson random measure denoted again by $M$ on $(0, \infty)^{3} \times \mathbb{N}$ with intensity $\mathrm{d} s m(\mathrm{~d} z) \mathrm{d} u \frac{(\lambda z)^{k}}{k !} \mathrm{e}^{-\lambda z} \sharp(\mathrm{d} k)$ for some $\lambda>0$ :

$$
\begin{aligned}
Y_{t}=Y_{0}- & a \int_{0}^{t} Y_{s} \mathrm{~d} s+\sqrt{2 \alpha} \int_{0}^{t} \int_{0}^{Y_{s}} W(\mathrm{~d} s, \mathrm{~d} u)+\int_{0}^{t} \int_{0}^{\infty} \int_{0}^{Y_{s-}} \int_{\mathbb{N}} z \tilde{M}(\mathrm{~d} s, \mathrm{~d} z, \mathrm{~d} u, \mathrm{~d} k) \\
=Y_{0}- & \phi^{\prime}(\lambda) \int_{0}^{t} Y_{s} \mathrm{~d} s+\sqrt{2 \alpha} \int_{0}^{t} \int_{0}^{Y_{s}} W(\mathrm{~d} s, \mathrm{~d} u) \\
& +\int_{0}^{t} \int_{0}^{\infty} \int_{0}^{Y_{s-}} z \tilde{M}^{0}(\mathrm{~d} s, \mathrm{~d} z, \mathrm{~d} u)+2 \alpha \lambda \int_{0}^{t} Y_{s} \mathrm{~d} s \\
& +\int_{0}^{t} \int_{0}^{\infty} \int_{0}^{Y_{s-}} z M^{1}(\mathrm{~d} s, \mathrm{~d} z, \mathrm{~d} u)+\int_{0}^{t} \int_{0}^{\infty} \int_{0}^{Y_{s-}} z M^{2}(\mathrm{~d} s, \mathrm{~d} z, \mathrm{~d} u),
\end{aligned}
$$

where $M^{0}(\mathrm{~d} s, \mathrm{~d} z, \mathrm{~d} u)=M(\mathrm{~d} s, \mathrm{~d} z, \mathrm{~d} u,\{k=0\})$ and

$$
M^{1}(\mathrm{~d} s, \mathrm{~d} z, \mathrm{~d} u)=M(\mathrm{~d} s, \mathrm{~d} z, \mathrm{~d} u,\{k=1\}), \quad M^{2}(\mathrm{~d} s, \mathrm{~d} z, \mathrm{~d} u)=M(\mathrm{~d} s, \mathrm{~d} z, \mathrm{~d} u,\{k \geq 2\}) .
$$

Recently, 9] gave another SDE-type description for one-dimensional CB-processes based on (1.4) with $a<0, \lambda \geq \lambda^{*}$, here $\lambda^{*}$ is the unique root of $\phi$ on $(0, \infty)$. One of the results in [9] shows that the last three integrals on the right-hand side of (1.4) are identified with the mass that immigrates from the skeleton construction. More precisely, the following stochastic equation system has a unique strong solution:

$$
\begin{aligned}
Y_{t}=Y_{0} & +\int_{0}^{t}\left(2 \alpha X_{s}-\phi^{\prime}(\lambda) Y_{s}\right) \mathrm{d} s+\sqrt{2 \alpha} \int_{0}^{t} \int_{0}^{Y_{s}} W(\mathrm{~d} s, \mathrm{~d} u) \\
& +\int_{0}^{t} \int_{0}^{\infty} \int_{0}^{Y_{s-}} z \tilde{M}^{0}(\mathrm{~d} s, \mathrm{~d} z, \mathrm{~d} u)+\int_{0}^{t} \int_{0}^{\infty} \int_{1}^{X_{s-}} z M^{3}(\mathrm{~d} s, \mathrm{~d} z, \mathrm{~d} k) \\
& +\int_{0}^{t} \int_{\mathbb{M}} \int_{1}^{X_{s-}} z_{1} M^{4}(\mathrm{~d} s, \mathrm{~d} z, \mathrm{~d} k) \\
X_{t}=X_{0} & +\int_{0}^{t} \int_{\mathbb{M}} \int_{1}^{X_{s-}}\left(z_{2}-1\right) M^{4}(\mathrm{~d} s, \mathrm{~d} z, \mathrm{~d} k)
\end{aligned}
$$

where $\mathbb{M}=\mathbb{R}_{+} \times \mathbb{N}, M^{3}(\mathrm{~d} s, \mathrm{~d} z, \mathrm{~d} k)$ is a Poisson random measure on $(0, \infty)^{2} \times\{\mathbb{N} \backslash\{0\}\}$ with intensity measure $\mathrm{d} s z \mathrm{e}^{-\lambda z} m(\mathrm{~d} z) \sharp(\mathrm{d} k), M^{4}(\mathrm{~d} s, \mathrm{~d} z, \mathrm{~d} k)$ is a Poisson random measure on $(0, \infty) \times$ $\mathbb{M} \times\{\mathbb{N} \backslash\{0\}\}$ with intensity measure $\phi^{\prime}(\lambda) \mathrm{d} s \eta_{z_{2}}\left(\mathrm{~d} z_{1}\right) p_{z_{2}} \sharp\left(\mathrm{d} z_{2}\right) \sharp(\mathrm{d} k)$, one can see the specific definitions of two distributions $\left(\eta_{k}\right)_{k \in \mathbb{N}}$ and $\left(p_{k}\right)_{k \in \mathbb{N}}$ in [9, p.1127] and we omitted here. The authors prove that for any $y \geq 0,\left\{Y_{t}: t \geq 0\right\}$ is a weak solution of (1.4) with initial value $Y_{0}=y$ if $X_{0}$ is Poisson distributed with parameter $\lambda y$. Moreover, (1.5)-(1.6) includes the prolific skeleton decomposition when $\lambda=\lambda^{*}$, see [2] for the properties of this special decomposition. And we refer to [10] for the similar construction of (1.5)-(1.6) in the setting of superprocesses.

Inspired by the formulations (1.5)-(1.6), the first objective of this paper is to construct a two-dimensional branching Markov process $\left\{\left(Y_{1}(t), Y_{2}(t)\right): t \geq 0\right\}$ taking values in $\mathbb{M}$ obtained 
as a unique strong solution to a more generalized stochastic equation system than (1.5)-(1.6), and the process is called the continuous time mixed state branching process (MSB-process). Indeed, the specific form of the stochastic equation system is as follows:

$$
\begin{aligned}
Y_{1}(t)=Y_{1}(0) & -a_{11} \int_{0}^{t} Y_{1}(s) \mathrm{d} s+\int_{0}^{t} \sqrt{2 \alpha Y_{1}(s)} \mathrm{d} B(s)+\int_{0}^{t} \int_{0}^{Y_{1}(s-)} \int_{\mathbb{M}} z_{1} \tilde{N}_{1}(\mathrm{~d} s, \mathrm{~d} u, \mathrm{~d} z) \\
& +a_{21} \int_{0}^{t} Y_{2}(s) \mathrm{d} s+\int_{0}^{t} \int_{0}^{Y_{2}(s-)} \int_{\mathbb{M}_{-1}} z_{1} N_{2}(\mathrm{~d} s, \mathrm{~d} u, \mathrm{~d} z), \\
Y_{2}(t)=Y_{2}(0) & +\int_{0}^{t} \int_{0}^{Y_{1}(s-)} \int_{\mathbb{M}} z_{2} N_{1}(\mathrm{~d} s, \mathrm{~d} u, \mathrm{~d} z)+\int_{0}^{t} \int_{0}^{Y_{2}(s-)} \int_{\mathbb{M}_{-1}} z_{2} N_{2}(\mathrm{~d} s, \mathrm{~d} u, \mathrm{~d} z),
\end{aligned}
$$

where $a_{21}, \alpha \geq 0, a_{11} \in \mathbb{R}, \mathbb{M}_{-1}=\mathbb{R}_{+} \times \mathbb{N}_{-1}, \mathbb{N}_{-1}=\mathbb{N} \cup\{-1\}, B$ is a standard Brownian motion, $N_{1}$ and $N_{2}$ are two Poisson random measures with intensity measures $\mathrm{d} s n_{1}(\mathrm{~d} z) \mathrm{d} u$ and $\mathrm{d} s n_{2}(\mathrm{~d} z) \mathrm{du}$, respectively, $n_{1}$ and $n_{2}$ are two Lévy measures satisfying some moment conditions. Intuitively, there exist interactions between $\left\{Y_{1}(t): t \geq 0\right\}$ and $\left\{Y_{2}(t): t \geq 0\right\}$, therefore (1.7)(1.8) obviously generalize the (1.5) -(1.6). We mention that the Brownian motion $B$ in (1.7) can be replaced by a space-time white noise $W$ and the process has the same law for any fixed initial value.

In the literature of the theory of branching processes, the rescaling approach plays a valuable role on establishing the connection among those branching processes, which leads to the second purpose of this paper and we establish two results. First, for a sequence of GW-processes $\left\{X_{k}(n): n \geq 0\right\}_{k \geq 1}$ and a positive sequence $\left\{\gamma_{k}\right\}_{k \geq 1}$, we show that on proper conditions $\left\{X_{k}\left(\left\lfloor\gamma_{k} t\right\rfloor\right): t \geq 0\right\}$ converges as $k \rightarrow \infty$ to a DB-process in distribution, where $\lfloor x\rfloor$ denotes the integral part of $x$. Second, for a sequence of two-type GW-processes $\left\{\left(Y_{k, 1}(n), Y_{k, 2}(n)\right)\right.$ : $n \in \mathbb{N}\}_{k \geq 1}$, we also prove $\left\{\left(k^{-1} Y_{k, 1}\left(\left\lfloor\gamma_{k} t\right\rfloor\right), Y_{k, 2}\left(\left\lfloor\gamma_{k} t\right\rfloor\right)\right): t \geq 0\right\}$ converges in distribution to a MSB-process under parallel conditions. The key of two limit theorems above is mainly inspired by [20, 21, 25].

The existence of the stationary distribution and ergodic rates are both important topics in the theory of Markov processes. A necessary and sufficient condition for the existence of the stationary distribution of one-dimensional CBI-processes was initiated by [29], see also [21] for a proof. The sufficient condition for the multi-type case can be found in 18. The strong Feller property and exponential ergodicity of such processes in the total variation distance were given in 22] by a coupling of CBI-processes constructed by the stochastic equation driven by time-space noises and Poisson random measures, see also [23]. In a recent work [24, the author considered the ergodicities and exponential ergodicities in Wasserstein and total variation distances of Dawson-Watanabe superprocesses with or without immigration, which clearly includes the multitype CBI-process case. After constructing the MSB-processes, we also want to study the ergodic theory of such processes and we prove the exponential ergodicity in the $L^{1}$-Wasserstein distance by establishing a upper bound estimates for the variations of the transition probabilities, which is inspired by the similar results of measure-valued branching processes in [24]. Moreover, by adding the immigration structures, we give a sufficient and necessary condition for the existence of the stationary distribution of MSB-processes with immigration (MSBI-processes).

The remainder of the paper is organized as follows. In section 2, we prove a weak convergence theorem from GW-processes to DB-processes. In section 3, we obtain the MSB-process arising in a limit theorem of rescaled two-type $\mathrm{GW}$-processes. In section 4 , we provide another construction 
of MSB-processes by stochastic equation systems. The analysis of distributions of jumps is given in section 5 . In section 6 , we study the estimates for the variations and the exponential ergodicity both in the $L^{1}$-Wasserstein distance $W_{1}$ for the transition semigroup of such processes. And we prove the existence of the stationary distribution of such processes with immigration in section 7 .

\section{The construction of DB-processes}

Let $\left\{p_{j}: j \in \mathbb{N}\right\}$ be a probability distribution on $\mathbb{N}$, and denote the generating function by $g(z)=\sum_{j=0}^{\infty} p_{j} z^{j}$ on $|z| \leq 1$. Let $u(z)=a(g(z)-z)$ for some $a>0$. A Markov process $\left\{X_{t}: t \geq 0\right\}$ with state space $\mathbb{N}$ is called a DB-process with branching rate $a>0$ and offspring distribution $\left\{p_{j}: j \in \mathbb{N}\right\}$ if its transition probabilities $Q_{i j}(t)$ satisfy

$$
\sum_{j=0}^{\infty} Q_{i j}(t) z^{j}=\left[\sum_{j=0}^{\infty} Q_{1 j}(t) z^{j}\right]^{i}, \quad i \in \mathbb{N}, \quad t \geq 0, \quad z \in[0,1],
$$

which implies the branching property of the process. Denote $F(z, t)=\sum_{k=0}^{\infty} Q_{1 k}(t) z^{k}$. Clearly, $F=(F(\cdot, t): t \geq 0)$ satisfies the semigroup property: $F(\cdot, t+s)=F(F(\cdot, t), s)$ for $t, s \geq 0$ and is the unique solution of the following differential equation:

$$
\frac{\partial}{\partial t} F(z, t)=u[F(z, t)], \quad F(z, 0)=z .
$$

We call $F$ the compound semigroup for the DB-process, we refer to [1, p.106-107] for more details.

We now provide a sufficient condition for the weak convergence of GW-processes to the DB-process. Assume that there exists a sequence of GW-processes $\left\{X_{k}(n): n \geq 0\right\}_{k \geq 1}$ with parameters $\left\{g_{k}\right\}_{k \geq 1}$, and let $\left\{\gamma_{k}\right\}_{k \geq 1}$ be a sequence of positive numbers. Denote the $n$-step transition probability for $\left\{X_{k}(n): n \geq 1\right\}$ by $Q_{k}^{n}$, let $\lfloor x\rfloor$ be the integral part of $x$. One can see that

$$
\sum_{j=0}^{\infty} Q_{k}^{\left\lfloor\gamma_{k} t\right\rfloor}(i, j) z^{j}=\left[g_{k}^{\circ\left\lfloor\gamma_{k} t\right\rfloor}(z)\right]^{i}:=\left(F_{k}(z, t)\right)^{i}, \quad i \in \mathbb{N}, \quad z \in[0,1], \quad t \geq 0,
$$

and

$$
\begin{aligned}
F_{k}(z, t) & =z+\sum_{i=1}^{\left\lfloor\gamma_{k} t\right\rfloor}\left(g_{k}^{\circ i}(z)-g_{k}^{\circ(i-1)}(z)\right) \\
& =z+\gamma_{k}-1 \sum_{i=1}^{\left\lfloor\gamma_{k} t\right\rfloor} U_{k}\left(F_{k}\left(z, \frac{i-1}{\gamma_{k}}\right)\right) \\
& =z+\int_{0}^{\frac{\left\lfloor\gamma_{k} t\right\rfloor}{\gamma_{k}}} U_{k}\left(F_{k}(z, r)\right) \mathrm{d} r
\end{aligned}
$$

where $g^{\circ n}(z)$ is defined by $g^{\circ n}(z)=g\left(g^{\circ(n-1)}(z)\right)$ successively with $g^{\circ 0}(z)=z$ and $U_{k}(z)=$ $\gamma_{k}\left(g_{k}(z)-z\right), 0 \leq z \leq 1$. For the convenience, we formulate the following conditions:

(A) $\gamma_{k} \rightarrow \infty$ as $k \rightarrow \infty$.

(B) The sequence $U_{k}(z)$ is uniformly Lipschitz on $[0,1]$, and converges to a continuous function $u(z)$ as $k \rightarrow \infty$. 
Proposition 2.1 (i) Suppose that $(\boldsymbol{A}, \boldsymbol{B})$ hold. Then the limit function of sequence $\left\{U_{k}(z)\right\}_{k \geq 1}$ has representation $u(z)=a(g(z)-z)$ as $k \rightarrow \infty$ for all $0 \leq z \leq 1$, where $a$ is a strictly positive constant, $g(z)$ is a generation function and $g^{\prime}(1-)<\infty$.

(ii) For any given $u(z)=a(g(z)-z)$, there exists a sequence of $\left\{U_{k}\right\}_{k \geq 1}$ such that $(\boldsymbol{A}, \boldsymbol{B})$ hold with $U_{k}(z) \rightarrow u(z)$.

Proof. (i) The desired result is a corollary of Proposition 3.1 (i) later. Indeed, it suffices to consider the offspring distribution corresponding to two-type GW-processes case satisfying $v_{k}(\{i, \cdot\}) \equiv 0$ for all $i \geq 1$.

(ii) For given $g(z)-z=\sum_{i=0}^{\infty} p_{i} z^{i}-z$ and $a>0$, set $\gamma_{k}=a k$ and

$$
p_{k i}= \begin{cases}p_{i} / k, & \text { if } i \neq 1 \\ \left(p_{1}-1\right) / k+1, & \text { if } i=1 .\end{cases}
$$

Define $U_{k}(z)=a k\left(\sum_{i=0}^{\infty} p_{k i} z^{i}-z\right)$, it is not hard to see that $U_{k}$ satisfies condition $(\mathbf{B})$, and converges to $u(z)$ for all $z \in[0,1]$.

Lemma 2.1 Suppose that $(\boldsymbol{A}, \boldsymbol{B})$ hold. Then there are constants $\lambda, N \geq 0$ such that $F_{k}(z, t) \in$ $\left[z^{\mathrm{e}^{\lambda t}}, 1\right]$ for every $t \geq 0, z \in[0,1]$ and $k \geq N$.

Proof. Let $b_{k}:=\gamma_{k}\left(g_{k}^{\prime}(1-)-1\right)$. Under condition (B), there exists $\lambda \geq 0$ such that $2\left|b_{k}\right| \leq \lambda$ for all $k \geq 1$. It is not hard to obtain

$$
\sum_{j=1}^{\infty} j Q_{k}^{\left\lfloor\gamma_{k} t\right\rfloor}(i, j)=i g_{k}^{\prime}(1-)^{\left\lfloor\gamma_{k} t\right\rfloor}=i\left(1+\frac{b_{k}}{\gamma_{k}}\right)^{\left\lfloor\gamma_{k} t\right\rfloor} .
$$

Since $\gamma_{k} \rightarrow \infty$ as $k \rightarrow \infty$, there is a $N \geq 1$ such that for all $k \geq N$

$$
0 \leq\left(1+\frac{b_{k}}{\gamma_{k}}\right)^{\frac{\gamma_{k}}{\lambda}} \leq\left(1+\frac{\lambda}{2 \gamma_{k}}\right)^{\frac{\gamma_{k}}{\lambda}} \leq \mathrm{e},
$$

then for $t \geq 0$ and $k \geq N$

$$
\sum_{j=1}^{\infty} j Q_{k}^{\left\lfloor\gamma_{k} t\right\rfloor}(i, j) \leq i \exp \left\{\frac{\lambda}{\gamma_{k}}\left\lfloor\gamma_{k} t\right\rfloor\right\} \leq i \mathrm{e}^{\lambda t} .
$$

We get the desired result by Jensen's inequality.

Lemma 2.2 Suppose that $(\boldsymbol{A}, \boldsymbol{B})$ hold. For any $c>0$, we have $F_{k}(z, t) \rightarrow$ some $F(z, t)$ uniformly on $\left[0, \mathrm{e}^{-c}\right] \times[0, c]$ as $k \rightarrow \infty$. And the limit function solves (2.2).

Proof. We may rewrite

$$
F_{k}(z, t)=z+\int_{0}^{t} U_{k}\left(F_{k}(z, r)\right) \mathrm{d} r-\left(t-\frac{\left\lfloor\gamma_{k} t\right\rfloor}{\gamma_{k}}\right) U_{k}\left(F_{k}\left(z, \frac{\left\lfloor\gamma_{k} t\right\rfloor}{\gamma_{k}}\right)\right) .
$$


By Proposition 2.1 and Lemma 2.1, for $\varepsilon \in(0,1]$, we can take $N \geq 1$ large enough such that

$$
\left|U_{k}(z)-u(z)\right| \leq \varepsilon, \quad \mathrm{e}^{-c \mathrm{e}^{\lambda c}} \leq z \leq 1, \quad k \geq N .
$$

Denote the last term on the right hand of equation (2.3) by $\varepsilon_{k}(t, z)$, then

$$
\left|\varepsilon_{k}(t, z)\right| \leq \gamma_{k}^{-1} M, \quad 0 \leq t \leq c, \quad 0 \leq z \leq \mathrm{e}^{-c},
$$

where $M=1+\sup _{\Theta}|u(z)|, \Theta=\left\{z: \mathrm{e}^{-c \mathrm{e}^{\lambda c}} \leq z \leq 1\right\}$. On the other hand, for $n \geq k \geq N$ we set

$$
K_{k, n}(t, z)=\sup _{0 \leq r \leq t}\left|F_{n}(z, r)-F_{k}(z, r)\right|
$$

it follows that

$$
K_{k, n}(t, z) \leq 2\left(\gamma_{k}^{-1} M+\varepsilon c\right)+\rho \int_{0}^{t} K_{k, n}(r, z) \mathrm{d} r, \quad 0 \leq t \leq c, \quad 0 \leq z \leq \mathrm{e}^{-c},
$$

where $\rho=\sup _{\Theta}\left|u^{\prime}(z)\right|$. By applying Gronwall inequality we obtain

$$
K_{k, n}(t, z) \leq 2\left(\gamma_{k}^{-1} M+\varepsilon c\right) \mathrm{e}^{\rho t}, \quad 0 \leq t \leq c, \quad 0 \leq z \leq \mathrm{e}^{-c},
$$

which follows that $F_{k}(z, t) \rightarrow$ some $F(z, t)$, and the limit function satisfies (2.2).

By (2.1), we see that the transition probabilities $Q=\left\{Q_{i j}(t): i, j \in \mathbb{N}, t \geq 0\right\}$ of the DB-process $\left\{X_{t}: t \geq 0\right\}$ can be determined by

$$
\sum_{j=0}^{\infty} Q_{i j}(t) z^{j}=(F(z, t))^{i}, \quad i \geq 1, t \geq 0 .
$$

Based on Proposition 2.1 and Lemma 2.2, by similar arguments of Theorem 2.9 in [23], it is not hard to see that the transition probabilities $Q$ of $\left\{X_{t}: t \geq 0\right\}$ is a limit of a sequence transition probabilities $\left\{Q_{k}^{\left\lfloor\gamma_{k} t\right\rfloor}(i, j): i, j \in \mathbb{N}, t \geq 0\right\}_{k \geq 1}$ associated with GW-processes in the sense of weak convergence under conditions $(\mathbf{A}, \mathbf{B})$, which indeed implies another construction of DB-processes by rescaling approach.

\section{The construction of MSB-processes}

For a two-type GW-process $\left\{Y(n)=\left(Y_{1}(n), Y_{2}(n)\right): n \in \mathbb{N}\right\}$, we define two corresponding generation functions

$$
g_{j}\left(s_{1}, s_{2}\right)=\sum_{i \in \mathbb{N}^{2}} p_{j}(i) s_{1}^{i_{1}} s_{2}^{i_{2}}, \quad p_{j}(i)=\mathbf{P}\left(Y(1)=i \mid Y(0)=\mathbf{e}_{j}\right), \quad j=1,2
$$

for $i=\left(i_{1}, i_{2}\right) \in \mathbb{N}^{2}$ and $s_{1}, s_{2} \in[0,1]$, where $\mathbf{e}_{1}=(1,0), \mathbf{e}_{2}=(0,1)$. Note that $g_{j}^{o n}=$ $g_{j}\left(g_{1}^{\circ(n-1)}, g_{2}^{\circ(n-1)}\right)$ for $j=1,2$ and $n \geq 1$. It is known that the one-step transition matrix $P(i, j)$ of $\{Y(n): n \geq 0\}$ is uniquely determined by

$$
\sum_{j \in \mathbb{N}^{2}} P(i, j) s_{1}^{j_{1}} s_{2}^{j_{2}}=\left[g_{1}\left(s_{1}, s_{2}\right)\right]^{i_{1}}\left[g_{2}\left(s_{1}, s_{2}\right)\right]^{i_{2}} .
$$


Let $\left\{Y_{k}(n)=\left(Y_{k, 1}(n), Y_{k, 2}(n)\right): n \in \mathbb{N}\right\}$ be a sequence of two-type $\mathrm{GW}$-processes corresponding to the $G_{k}=\left(g_{k, 1}, g_{k, 2}\right)$, and $\left\{\gamma_{k}\right\}$ be a sequence of positive numbers. Denote the $n$-step transition probability for $\left\{\left(k^{-1} Y_{k, 1}(n), Y_{k, 2}(n)\right): n \geq 0\right\}$ by $P_{k}^{n}$, it is not hard to see that for $t \geq 0$ and $\lambda \in \mathbb{R}_{+}^{2}$

$$
\int_{\mathbb{M}_{k}} \mathrm{e}^{-\langle\lambda, y\rangle} P_{k}^{\left\lfloor\gamma_{k} t\right\rfloor}(x, \mathrm{~d} y)=\left[g_{k, 1}^{\circ\left\lfloor\gamma_{k} t\right\rfloor}\left(\mathrm{e}^{-\lambda_{1} / k}, \mathrm{e}^{-\lambda_{2}}\right)\right]^{k x_{1}}\left[g_{k, 2}^{\circ\left\lfloor\gamma_{k} t\right\rfloor}\left(\mathrm{e}^{-\lambda_{1} / k}, \mathrm{e}^{-\lambda_{2}}\right)\right]^{x_{2}}, \quad x \in \mathbb{M}_{k},
$$

where $\mathbb{M}_{k}:=\left\{(i / k, j):(i, j) \in \mathbb{N}^{2}\right\}$. For $\lambda=\left(\lambda_{1}, \lambda_{2}\right) \in \mathbb{R}_{+}^{2}$, we define a vector function $V_{k}(t, \lambda)=\left(V_{k, 1}(t, \lambda), V_{k, 2}(t, \lambda)\right)$ with

$$
V_{k, 1}(t, \lambda)=-k \log g_{k, 1}^{\circ\left\lfloor\gamma_{k} t\right\rfloor}\left(\mathrm{e}^{-\lambda_{1} / k}, \mathrm{e}^{-\lambda_{2}}\right), \quad V_{k, 2}(t, \lambda)=-\log g_{k, 2}^{\circ\left\lfloor\gamma_{k} t\right\rfloor}\left(\mathrm{e}^{-\lambda_{1} / k}, \mathrm{e}^{-\lambda_{2}}\right),
$$

then we can rewrite

$$
\int_{\mathbb{M}_{k}} \mathrm{e}^{-\langle\lambda, y\rangle} P_{k}^{\left\lfloor\gamma_{k} t\right\rfloor}(x, \mathrm{~d} y)=\exp \left\{-\left\langle x, V_{k}(t, \lambda)\right\rangle\right\}, \quad x \in \mathbb{M}_{k}
$$

and $V_{k}(t, \lambda)$ satisfies the following equation

$$
V_{k, 1}(t, \lambda)=\lambda_{1}-\int_{0}^{\frac{\left\lfloor\gamma_{k} t\right\rfloor}{\gamma_{k}}} \bar{\Phi}_{k, 1}\left(V_{k}(s, \lambda)\right) \mathrm{d} s, \quad V_{k, 2}(t, \lambda)=\lambda_{2}-\int_{0}^{\frac{\left\lfloor\gamma_{k} t\right\rfloor}{\gamma_{k}}} \bar{\Phi}_{k, 2}\left(V_{k}(s, \lambda)\right) \mathrm{d} s,
$$

where two functions $\bar{\Phi}_{k, 1}$ and $\bar{\Phi}_{k, 2}$ are defined on $\mathbb{R}_{+}^{2}$ taking the form of

$$
\begin{aligned}
& \bar{\Phi}_{k, 1}\left(\lambda_{1}, \lambda_{2}\right)=k \gamma_{k} \log \left[1-\left(k \gamma_{k}\right)^{-1} \Phi_{k, 1}\left(\lambda_{1}, \lambda_{2}\right) \mathrm{e}^{\frac{\lambda_{1}}{k}}\right], \\
& \Phi_{k, 1}\left(\lambda_{1}, \lambda_{2}\right)=k \gamma_{k}\left[\mathrm{e}^{-\frac{\lambda_{1}}{k}}-g_{k, 1}\left(\mathrm{e}^{-\frac{\lambda_{1}}{k}}, \mathrm{e}^{-\lambda_{2}}\right)\right], \\
& \bar{\Phi}_{k, 2}\left(\lambda_{1}, \lambda_{2}\right)=\gamma_{k} \log \left[1-\gamma_{k}^{-1} \Phi_{k, 2}\left(\lambda_{1}, \lambda_{2}\right) \mathrm{e}^{\lambda_{2}}\right], \\
& \Phi_{k, 2}\left(\lambda_{1}, \lambda_{2}\right)=\gamma_{k}\left[\mathrm{e}^{-\lambda_{2}}-g_{k, 2}\left(\mathrm{e}^{-\frac{\lambda_{1}}{k}}, \mathrm{e}^{-\lambda_{2}}\right)\right] .
\end{aligned}
$$

We further define two functions $\Phi_{1}$ and $\Phi_{2}$ on $[0, \infty)^{2}$ :

$$
\begin{aligned}
& \Phi_{1}\left(\lambda_{1}, \lambda_{2}\right)=-a_{11} \lambda_{1}-\alpha \lambda_{1}^{2}-\int_{\mathbb{M}}\left(\mathrm{e}^{-\langle\lambda, z\rangle}-1+\lambda_{1} z_{1}\right) n_{1}(\mathrm{~d} z), \\
& \Phi_{2}\left(\lambda_{1}, \lambda_{2}\right)=a_{21} \lambda_{1}+\int_{\mathbb{M}_{-1}}\left(1-\mathrm{e}^{-\langle\lambda, z\rangle}\right) n_{2}(\mathrm{~d} z),
\end{aligned}
$$

where $a_{11}$ is a constant, $a_{21}, \alpha \geq 0, n_{1}$ and $n_{2}$ are $\sigma$ finite measures on $\mathbb{M}$ and $\mathbb{M}_{-1}$, supported by $\mathbb{M} \backslash\{\mathbf{0}\}$ and $\mathbb{M}_{-1} \backslash\{\mathbf{0}\}$, respectively, such that $n_{2}\left(\mathbb{R}_{+} \times\{-1\}\right)<\infty$ and

$$
\int_{\mathbb{M}}\left(z_{1} \wedge z_{1}^{2}+z_{2}\right) n_{1}(\mathrm{~d} z)+\int_{\mathbb{M}_{-1}}\left(z_{1}+z_{2}\right) n_{2}(\mathrm{~d} z)<\infty .
$$

For convenience, let us consider the following conditions:

(A) $\gamma_{k} \rightarrow \infty$.

(C) The sequence $\left\{\Phi_{k, 1}\left(\lambda_{1}, \lambda_{2}\right)\right\}_{k \geq 1}$ is uniformly Lipschitz in $\left(\lambda_{1}, \lambda_{2}\right)$ on each bounded rectangle, and converges to a continuous function as $k \rightarrow \infty$.

(D) The sequence $\left\{\mathrm{e}^{\lambda_{2}} \Phi_{k, 2}\left(\lambda_{1}, \lambda_{2}\right)\right\}_{k \geq 1}$ is uniformly Lipschitz in $\left(\lambda_{1}, \lambda_{2}\right)$ on each bounded rectangle, and converges to a continuous function as $k \rightarrow \infty$. 
Proposition 3.1 (i) Assume that $(\boldsymbol{A}, \boldsymbol{C}, \boldsymbol{D})$ hold. Then the limit function $\Phi_{1}\left(\lambda_{1}, \lambda_{2}\right)$ has the representation (3.3), and the limit $\Phi_{2}\left(\lambda_{1}, \lambda_{2}\right)$ of has the representation (3.4);

(ii) For any $\Phi_{1}$ and $\Phi_{2}$ given by (3.3) and (3.4), there are sequences $\left\{\gamma_{k}\right\}$ and $\left\{\left(g_{k, 1}, g_{k, 2}\right)\right\}$ as above such that $(\boldsymbol{A}, \boldsymbol{C}, \boldsymbol{D})$ hold with $\Phi_{k, 1}\left(\lambda_{1}, \lambda_{2}\right) \rightarrow \Phi_{1}\left(\lambda_{1}, \lambda_{2}\right)$ and $\mathrm{e}^{\lambda_{2}} \Phi_{k, 2}\left(\lambda_{1}, \lambda_{2}\right) \rightarrow \Phi_{2}\left(\lambda_{1}, \lambda_{2}\right)$ for all $\left(\lambda_{1}, \lambda_{2}\right) \in \mathbb{R}_{+}^{2}$ as $k \rightarrow \infty$.

Proof. (i) We first prove the representation result for $\Phi_{2}$, which is inspired by [25].

(1) Fix $k \geq 1$. Let $\mathbb{M}_{-1, k}=\left\{(i / k, j-1):\{i, j\} \in \mathbb{N}^{2}\right\}, \rho_{k}$ be the measure defined by:

$$
\rho_{k}(\cdot)=\gamma_{k} \sum_{i, j=0}^{\infty} v_{k}(\{i, j\}) \delta_{(i / k, j-1)}(\cdot),
$$

where $v_{k}$ be the probability measure on $\mathbb{N}^{2}$ corresponding to $g_{k, 2}$, then $\rho_{k}$ is a finite measure on $\mathbb{M}_{-1, k}$. Let $l(z)=\left(z_{1}+\left|z_{2}\right|\right) \wedge 1, \varrho_{k}=\int_{\mathbb{M}_{-1}} l(z) \rho_{k}(\mathrm{~d} z)$. If $\varrho_{k}>0$, define $P_{k}(\mathrm{~d} z)=$ $\left(l(z) / \varrho_{k}\right) \rho_{k}(\mathrm{~d} z)$, if $\varrho_{k}=0$, we let $P_{k}(\cdot)$ be the Dirac measure at some point $z_{0} \in \mathbb{M}_{-1, k} \backslash\{0\}$. In both cases, we have that $P_{k}(\cdot)$ is a probability measure on $\mathbb{M}_{-1}$ and

$$
\mathrm{e}^{\lambda_{2}} \Phi_{k, 2}\left(\lambda_{1}, \lambda_{2}\right)=\varrho_{k} \int_{\mathbb{M}_{-1} \backslash\{0\}}\left(1-\mathrm{e}^{-\langle\lambda, z\rangle}\right) l(z)^{-1} P_{k}(\mathrm{~d} z) .
$$

(2) Let $\mathbb{M}_{-1}^{\Delta}=\mathbb{M}_{-1} \cup\{\Delta\}$ be the one-point compactification of $\mathbb{M}_{-1}$. Then $\left\{P_{k}\right\}$ is the sequence of probability measures on $\mathbb{M}_{-1}^{\Delta}$, so it is relatively compact. Choose any subsequence denoted again by $\left\{P_{k}\right\}$, which converges to a probability $P$ on $\mathbb{M}_{-1}^{\Delta}$. Let $E=\{\varepsilon \mid P(\|z\|=\varepsilon)=0\}$, for $\varepsilon \in E$, we define a compact space of $\mathbb{M}_{-1}^{\Delta}$ by $Q:=\left\{z \in \mathbb{M}_{-1}^{\Delta},\|z\| \leq \varepsilon\right\}$. We have

$$
\mathrm{e}^{\lambda_{2}} \Phi_{k, 2}\left(\lambda_{1}, \lambda_{2}\right)=\varrho_{k} a_{k, \varepsilon}^{(1)} \lambda_{1}+\varrho_{k} a_{k, \varepsilon}^{(2)} \lambda_{2}-\varrho_{k}\left(I_{k, \varepsilon}+J_{k, \varepsilon}\right),
$$

where

$$
\begin{aligned}
a_{k, \varepsilon}^{(i)} & =\int_{Q \backslash\{0\}} \chi\left(z_{i}\right) l(z)^{-1} P_{k}(\mathrm{~d} z), \quad i=1,2, \quad \chi\left(z_{i}\right)=\left(1 \wedge z_{i}\right) \vee(-1), \\
J_{k, \varepsilon} & =\int_{\mathbb{M}_{-1} \backslash Q}\left(\mathrm{e}^{-\langle\lambda, z\rangle}-1\right) l(z)^{-1} P_{k}(\mathrm{~d} z), \\
I_{k, \varepsilon} & =\int_{Q \backslash\{0\}}\left(\mathrm{e}^{-\langle\lambda, z\rangle}-1+\chi\left(z_{1}\right) \lambda_{1}+\chi\left(z_{2}\right) \lambda_{2}\right) l(z)^{-1} P_{k}(\mathrm{~d} z) .
\end{aligned}
$$

It is not hard to see that $a_{k, \varepsilon}^{(2)}=0, \lim _{E \ni \varepsilon \downarrow 0} \lim _{k \rightarrow \infty} I_{k, \varepsilon}=0$.

(3) Fix $\varepsilon \in E, 0<\varepsilon<1$. If $\lim \inf _{k \rightarrow \infty} \varrho_{k}=0$, then $\Phi_{2}=0$. If $\lim _{k \rightarrow \infty} \inf _{k} \varrho_{k}>0$, then there exists a subsequence denoted again by $\left\{\varrho_{k}\right\}$ converges to $\varrho \in(0, \infty]$. We can prove that $P(\{\Delta\})=0$. Actually,

$$
\int_{\mathbb{M}_{-1} \backslash Q} \mathrm{e}^{-\langle\lambda, z\rangle} l(z)^{-1} P_{k}(\mathrm{~d} z) \rightarrow \text { some } L(\lambda),
$$

where $L(\lambda)$ is a continuous function. Since $l(z)^{-1} \rightarrow 1$ as $z \rightarrow \Delta$ and is the continuous function of $z \in \mathbb{M}_{-1}^{\Delta} \backslash Q$, it follows from monotone convergence theorem that

$$
\begin{aligned}
& \int_{\mathbb{M}_{-1} \backslash Q} l(z)^{-1} P(\mathrm{~d} z)=\lim _{k \rightarrow \infty} \int_{\mathbb{M}_{-1} \backslash\{0\}} l(z)^{-1} P_{k}(\mathrm{~d} z)=L(0), \\
& \int_{\mathbb{M}_{-1}^{\Delta} \backslash Q} l(z)^{-1} P(\mathrm{~d} z)=\lim _{n \rightarrow \infty} \int_{\mathbb{M}_{-1} \backslash Q} \mathrm{e}^{-\left\langle\frac{1}{n}, z\right\rangle} l(z)^{-1} P(\mathrm{~d} z)=\lim _{n \rightarrow \infty} L\left(\frac{1}{n}\right)=L(0) .
\end{aligned}
$$


Define $v(\{0\})=0$, and $v(\mathrm{~d} z)=l(z)^{-1} P(\mathrm{~d} z)$ on $\left\{z \in \mathbb{M}_{-1}:\|z\|>0\right\}$, we have

$$
\lim _{E \ni \varepsilon \downarrow 0} \lim _{k \rightarrow \infty} J_{k, \varepsilon}=\int_{\mathbb{M}_{-1}}\left(\mathrm{e}^{-\langle\lambda, z\rangle}-1\right) v(\mathrm{~d} z), \quad \lim _{E \ni \varepsilon \downarrow 0} \lim _{k \rightarrow \infty} a_{k, \varepsilon}^{(1)}:=a^{(1)} \geq 0 .
$$

Based on calculations above, we obtain that

$$
\frac{1}{\varrho} \Phi_{2}\left(\lambda_{1}, \lambda_{2}\right)=a^{(1)} \lambda_{1}+\int_{\mathbb{M}_{-1}}\left(1-\mathrm{e}^{-\langle\lambda, z\rangle}\right) v(\mathrm{~d} z) .
$$

(4) Now we need to verify that $1 / \varrho>0$. If not, the r.h.s of (3.7) is the zero function. Assume $v(\mathbb{M} \backslash\{0\})=0$, then $P(\{0\})=1$, and by the representations of $a_{k, \varepsilon}^{(i)}$, we can have $a^{(1)}=$ $\lim _{E \ni \varepsilon \downarrow 0} P(\|z\| \leq \varepsilon)=1$, but it follows from the representation of Lévy-Khintchine type functions that the parameters are unique, therefore $1 / \varrho=0$ is impossible. Let $n_{2}(\cdot)=\varrho v(\cdot), a_{21}=\varrho a^{(1)}$, then we can rewrite $\Phi_{2}$ as follows:

$$
\Phi_{2}\left(\lambda_{1}, \lambda_{2}\right)=a_{21} \lambda_{1}+\int_{\mathbb{M}_{-1}}\left(1-\mathrm{e}^{-\langle\lambda, z\rangle}\right) n_{2}(\mathrm{~d} z) .
$$

By applying the monotone convergence theorem, we get

$$
\frac{\partial \Phi_{2}}{\partial \lambda_{1}}(0+, 0)=a_{21}+\int_{\mathbb{M}_{-1}} z_{1} n_{2}(\mathrm{~d} z), \quad \frac{\partial \Phi_{2}}{\partial \lambda_{2}}(0,0+)=\int_{\mathbb{M}_{-1}} z_{2} n_{2}(\mathrm{~d} z) .
$$

From the fact that $\Phi_{2}$ is locally Lipschitz, we have $\int_{\mathbb{M}_{-1}}\left(z_{1}+z_{2}\right) n_{2}(\mathrm{~d} z)<\infty$ and $n_{2}\left(\mathbb{R}_{+} \times\right.$ $\{-1\})<\infty$.

Now we treat with $\Phi_{k, 1}$. Fix $k \geq 1$, let $W=[-1, \infty) \times \mathbb{N}, W_{k}=\left\{((i-1) / k, j):(i, j) \in \mathbb{N}^{2}\right\}$. We define $\tilde{\rho}_{k}$ by:

$$
\tilde{\rho}_{k}(\cdot)=k \gamma_{k} \sum_{i, j=0}^{\infty} \tilde{v}_{k}(\{i, j\}) \delta_{\left(\frac{i-1}{k}, j\right)}(\cdot),
$$

where $\tilde{v}_{k}$ is the probability measure on $\mathbb{N}^{2}$ corresponding to $g_{k, 1}$. Let $\tilde{l}(z)=\left(z_{1}^{2}+z_{2}\right) \wedge 1$, we have

$$
\mathrm{e}^{\lambda_{1} / k} \Phi_{k, 1}\left(\lambda_{1}, \lambda_{2}\right)=\tilde{\beta}_{k, 1} \lambda_{1}+\tilde{\varrho}_{k} \tilde{a}_{k, \varepsilon}^{(2)} \lambda_{2}-\tilde{\varrho}_{k}\left(\tilde{a}_{k, \varepsilon}^{(1)} \lambda_{1}^{2}+\tilde{I}_{k, \varepsilon}+\tilde{J}_{k, \varepsilon}\right)
$$

where

$$
\begin{aligned}
& \tilde{\beta}_{k, 1}=\int_{W} \chi\left(z_{1}\right) \tilde{\rho}_{k}(\mathrm{~d} z), \quad \tilde{a}_{k, \varepsilon}^{(1)}=\frac{1}{2} \int_{Q \backslash\{0\}} \chi\left(z_{1}\right)^{2} \tilde{l}(z)^{-1} \tilde{P}_{k}(\mathrm{~d} z), \\
& \tilde{a}_{k, \varepsilon}^{(2)}=\int_{Q \backslash\{0\}} \chi\left(u_{2}\right) \tilde{l}(u)^{-1} \tilde{P}_{k}(\mathrm{~d} u), \quad h(u, \lambda)=\mathrm{e}^{-\langle\lambda, z\rangle}-1+\chi\left(z_{1}\right) \lambda_{1}, \\
& \tilde{J}_{k, \varepsilon}=\int_{W \backslash Q} h(z, \lambda) \tilde{l}(z)^{-1} \tilde{P}_{k}(\mathrm{~d} z), \\
& \tilde{I}_{k, \varepsilon}=\int_{Q \backslash\{0\}}\left(h(z, \lambda)+\chi\left(z_{2}\right) \lambda_{2}-\frac{1}{2} \chi\left(z_{1}\right)^{2} \lambda_{1}^{2}\right) \tilde{l}(z)^{-1} \tilde{P}_{k}(\mathrm{~d} z) .
\end{aligned}
$$

Note that $\tilde{a}_{k, \varepsilon}^{(2)}=0$. By a similar argument in [25], we have

$$
\Phi_{1}\left(\lambda_{1}, \lambda_{2}\right)=-a_{11} \lambda_{1}-\alpha \lambda_{1}^{2}-\int_{\mathbb{M}}\left(\mathrm{e}^{-\langle\lambda, z\rangle}-1+\lambda_{1} z_{1}\right) n_{1}(\mathrm{~d} z),
$$


and $n_{1}$ satisfies

$$
\int_{\mathbb{M}}\left(z_{1} \wedge z_{1}^{2}+z_{2}\right) n_{1}(\mathrm{~d} z)<\infty
$$

(ii) Given function by (3.4), we set $\bar{D}_{k}=\left\{z \in \mathbb{M}_{-1}: z_{1}>\frac{1}{\sqrt{k}}\right\}, \bar{\gamma}_{k, 1}=a_{21} k, \bar{\gamma}_{k, 2}=n_{2}\left(\bar{D}_{k}\right)$, $\bar{g}_{k, 1}\left(x_{1}, x_{2}\right)=x_{1} x_{2}$ and $\bar{g}_{k, 2}\left(x_{1}, x_{2}\right)=\bar{\gamma}_{k, 2}^{-1} \int_{\bar{D}_{k}} x_{1}^{k z_{1}} x_{2}^{\left(z_{2}+1\right)} n_{2}(\mathrm{~d} z)$. Then if we set sequences $\bar{\gamma}_{k}=$ $\bar{\gamma}_{k, 1}+\bar{\gamma}_{k, 2}$ and $\overline{\mathrm{g}}_{k}^{(2)}=\bar{\gamma}_{k}^{-1}\left(\bar{\gamma}_{k, 1} \overline{\mathrm{g}}_{k, 1}+\bar{\gamma}_{k, 2} \overline{\mathrm{g}}_{k, 2}\right)$, we find the sequences $\left\{\bar{\gamma}_{k}\right\}$ and $\left\{\overline{\mathrm{g}}_{k}^{(2)}\right\}$ such that conditions $(\mathbf{A}, \mathbf{D})$ hold with $\mathrm{e}^{\lambda_{2}} \Phi_{k, 2}\left(\lambda_{1}, \lambda_{2}\right) \rightarrow \Phi_{2}\left(\lambda_{1}, \lambda_{2}\right)$ for all $\left(\lambda_{1}, \lambda_{2}\right) \in \mathbb{R}_{+}^{2}$ as $k \rightarrow \infty$.

On the other hand, given function by (3.3). Firstly, we set $\tilde{\gamma}_{k, 1}=a_{11}, \tilde{g}_{k, 1}\left(x_{1}, x_{2}\right)=1$. Secondly, set $\tilde{\gamma}_{k, 2}=(2 \alpha+1) k, \tilde{\mathrm{g}}_{k, 2}\left(x_{1}, x_{2}\right)=x_{1}+\frac{\alpha}{2 \alpha+1}\left(1-x_{1}\right)^{2}$. Thirdly, let $\tilde{D}_{k}=\{z \in \mathbb{M}$ : $\left.z_{1}>\frac{1}{\sqrt{k}}, z_{2}>\frac{1}{\sqrt{k}}\right\}$, and $\sigma_{k}=\int_{\tilde{D}_{k}}\left(z_{1}-\frac{1}{k}\right) n_{1}(\mathrm{~d} z)$, set the sequences

$$
\begin{aligned}
& \tilde{\gamma}_{k, 3}=\sigma_{k}+\frac{1}{k} n_{1}\left(\tilde{D}_{k}\right)+1 \\
& \tilde{g}_{k, 3}\left(x_{1}, x_{2}\right)=\frac{1}{k \tilde{\gamma}_{k, 3}} \int_{\tilde{D}_{k}} x_{1}^{k z_{1}} x_{2}^{z_{2}} n_{1}(\mathrm{~d} z)+\frac{\sigma_{k}+1}{\tilde{\gamma}_{k, 3}}\left(x_{1}+\frac{\sigma_{k}}{\sigma_{k}+1}\left(1-x_{1}\right)\right) .
\end{aligned}
$$

Fourthly, if we let $\tilde{\gamma}_{k}=\tilde{\gamma}_{k, 1}+\tilde{\gamma}_{k, 2}+\tilde{\gamma}_{k, 3}$ and $\tilde{\mathrm{g}}_{k}^{(1)}=\tilde{\gamma}_{k}^{-1}\left(\tilde{\gamma}_{k, 1} \tilde{\mathrm{g}}_{k, 1}+\tilde{\gamma}_{k, 2} \tilde{\mathrm{g}}_{k, 2}+\tilde{\gamma}_{k, 3} \tilde{\mathrm{g}}_{k, 3}\right)$, we find that the sequences $\left\{\tilde{\gamma}_{k}\right\}$ and $\left\{\tilde{\mathrm{g}}_{k}^{(1)}\right\}$ such that conditions $(\mathbf{A}, \mathbf{C})$ hold with $\Phi_{k, 1}\left(\lambda_{1}, \lambda_{2}\right) \rightarrow \Phi_{1}\left(\lambda_{1}, \lambda_{2}\right)$ for all $\left(\lambda_{1}, \lambda_{2}\right) \in \mathbb{R}_{+}^{2}$ as $k \rightarrow \infty$.

Now let $\gamma_{k}=\tilde{\gamma}_{k}+\bar{\gamma}_{k}$ and

$$
\begin{aligned}
& \mathrm{g}_{k, 1}\left(x_{1}, x_{2}\right)=\gamma_{k}^{-1}\left(\tilde{\gamma}_{k} \tilde{\mathrm{g}}_{k}^{(1)}\left(x_{1}, x_{2}\right)+\bar{\gamma}_{k} x_{1}\right), \\
& \mathrm{g}_{k, 2}\left(x_{1}, x_{2}\right)=\gamma_{k}^{-1}\left(\bar{\gamma}_{k} \overline{\mathrm{g}}_{k}^{(2)}\left(x_{1}, x_{2}\right)+\tilde{\gamma}_{k} x_{2}\right),
\end{aligned}
$$

we finally find the common sequences $\left\{\gamma_{k}\right\}$ and $\left\{\left(\mathrm{g}_{k, 1}, \mathrm{~g}_{k, 2}\right)\right\}$ such that conditions (A,C,D) hold with $\Phi_{k, 1}\left(\lambda_{1}, \lambda_{2}\right) \rightarrow \Phi_{1}\left(\lambda_{1}, \lambda_{2}\right)$ and $\mathrm{e}^{\lambda_{2}} \Phi_{k, 2}\left(\lambda_{1}, \lambda_{2}\right) \rightarrow \Phi_{2}\left(\lambda_{1}, \lambda_{2}\right)$ for all $\left(\lambda_{1}, \lambda_{2}\right) \in \mathbb{R}_{+}^{2}$ as $k \rightarrow \infty$.

Proposition 3.2 Assume that $(\boldsymbol{A}, \boldsymbol{C}, \boldsymbol{D})$ hold. Then for any $a \geq 0$ we have $V_{k}(t, \lambda) \rightarrow$ some $V(t, \lambda)$ uniformly on $[0, a]^{3}$ as $k \rightarrow \infty$, and the limit function solves the following integral equations:

$$
V_{1}(t, \lambda)=\lambda_{1}+\int_{0}^{t} \Phi_{1}(V(s, \lambda)) \mathrm{d} s, \quad V_{2}(t, \lambda)=\lambda_{2}+\int_{0}^{t} \Phi_{2}(V(s, \lambda)) \mathrm{d} s .
$$

Moreover, suppose that $\left(\Phi_{1}, \Phi_{2}\right)$ is given by (3.3)-(3.4). Then for any $\lambda \in \mathbb{R}_{+}^{2}$, the solution $t \mapsto V(t, \lambda)$ of (3.8) is unique, and the solution satisfies the semigroup property:

$$
V(r+t, \lambda)=V(r, V(t, \lambda)), \quad r, t \geq 0 .
$$

Proof. By a similar argument of Lemma 2.2 in [23, it follows from Proposition 3.1 that $\bar{\Phi}_{k, 1} \rightarrow$ $-\Phi_{1}$ and $\bar{\Phi}_{k, 2} \rightarrow-\Phi_{2}$ uniformly on each bounded rectangle, respectively, as $k \rightarrow \infty$. We can rewrite

$$
\begin{aligned}
& V_{k, 1}(t, \lambda)=\lambda_{1}-\int_{0}^{t} \bar{\Phi}_{k, 1}\left(V_{k}(s, \lambda)\right) \mathrm{d} s+\varepsilon_{k, 1}(t, \lambda), \\
& V_{k, 2}(t, \lambda)=\lambda_{2}-\int_{0}^{t} \bar{\Phi}_{k, 2}\left(V_{k}(s, \lambda)\right) \mathrm{d} s+\varepsilon_{k, 2}(t, \lambda),
\end{aligned}
$$


where

$$
\varepsilon_{k, i}(t, \lambda)=-\left(t-\gamma_{k}^{-1}\left\lfloor\gamma_{k} t\right\rfloor\right) \bar{\Phi}_{k, i}\left(V_{k}\left(\gamma_{k}^{-1}\left\lfloor\gamma_{k} t\right\rfloor, \lambda\right)\right), \quad i=1,2
$$

For $\theta \in \mathbb{R}_{+}^{2}$, it is not hard to obtain that

$$
\begin{aligned}
& \int_{\mathbb{M}_{k}}\left(\theta_{1} y_{1}+\theta_{2} y_{2}\right) P_{k}^{\left\lfloor\gamma_{k} t\right\rfloor}(x, \mathrm{~d} y) \\
& =\left(k^{-1} \theta_{1} g_{k, 1,(1)}^{\prime}(1)+\theta_{2} g_{k, 1,(2)}^{\prime}(1), k^{-1} \theta_{1} g_{k, 2,(1)}^{\prime}(1)+\theta_{2} g_{k, 2,(2)}^{\prime}(1)\right) A_{k}^{\left(\left\lfloor\gamma_{k} t\right\rfloor-1\right)}\left(k x_{1}, x_{2}\right)^{\top} \\
& =\left(k^{-1}\left[\theta_{1}\left(\gamma_{k}^{-1} \Phi_{k, 1,(1)}^{\prime}(0)+1\right)+\theta_{2} \gamma_{k}^{-1} \Phi_{k, 1,(2)}^{\prime}(0)\right], \theta_{1} \gamma_{k}^{-1} \Phi_{k, 2,(1)}^{\prime}(0)\right. \\
& \left.\quad+\theta_{2}\left(\gamma_{k}^{-1} \Phi_{k, 1,(2)}^{\prime}(0)+1\right)\right) A_{k}^{\left(\left\lfloor\gamma_{k} t\right\rfloor-1\right)}\left(k x_{1}, x_{2}\right)^{\top},
\end{aligned}
$$

where

$$
A_{k}=\left(\begin{array}{cc}
g_{k, 1,(1)}^{\prime}(1) & g_{k, 2,(1)}^{\prime}(1) \\
g_{k, 1,(2)}^{\prime}(1) & g_{k, 2,(2)}^{\prime}(1)
\end{array}\right), \quad A_{k}^{(n)}=A_{k} \times A_{k}^{(n-1)}
$$

and $g_{k, 1,(i)}^{\prime}, g_{k, 2,(i)}^{\prime}, \Phi_{k, 1,(i)}^{\prime}$ and $\Phi_{k, 2,(i)}^{\prime}$ denote the derivative with respect to $\lambda_{i}, i=1,2$, respectively. By assumption (C,D), there exists $B \geq 0$ such that $\left|\Phi_{k, i,(j)}^{\prime}\right| \leq B$ for all $i, j \in\{1,2\}$ and $k \geq 1$. It follows from Jensen's inequality that

$$
\left\langle x, V_{k}(t, \theta)\right\rangle \leq\left(\gamma_{k}^{-1} B+1\right)\left(\theta_{1}+\theta_{2}\right)\left(k^{-1}, 1\right) A_{k}^{\left(\left\lfloor\gamma_{k} t\right\rfloor-1\right)}\left(k x_{1}, x_{2}\right)^{\top},
$$

then

$$
\begin{aligned}
\left\|V_{k}(t, \theta)\right\| & =\sup _{\|x\|=1}\left\langle x, V_{k}(t, \theta)\right\rangle \leq\left(\gamma_{k}^{-1} B+1\right)\left(\theta_{1}+\theta_{2}\right) \sup _{\|x\|=1}\left(k^{-1}, 1\right) B_{k}^{\left(\left\lfloor\gamma_{k} t\right\rfloor-1\right)}\left(k x_{1}, x_{2}\right)^{\top} \\
& \leq\left(2 \gamma_{k}^{-1} B+1\right)^{\left\lfloor\gamma_{k} t\right\rfloor} \sqrt{2}\left(\theta_{1}+\theta_{2}\right),
\end{aligned}
$$

where

$$
B_{k}=\left(\begin{array}{cc}
\gamma_{k}^{-1} B+1 & \gamma_{k}^{-1} B k \\
\left(k \gamma_{k}\right)^{-1} B & \gamma_{k}^{-1} B+1
\end{array}\right),
$$

by a modification of the proof of Lemma 2.6 and Theorem 2.7 in [23], we get (3.8). For given $\left(\Phi_{1}, \Phi_{2}\right)$ by (3.3)-(3.4), it follows from Proposition 3.1 (ii) that there is a sequence $\left\{\Phi_{k, 1}, \Phi_{k, 2}\right\}$ satisfying (A,C,D), let a sequence $\left\{V_{k}\right\}$ be given by (3.1) and (3.2), the existence of the solution is immediate. The uniqueness of the solution follows by Gronwall's inequality, and the semigroup property follows from the uniqueness of the solution.

Proposition 3.3 Suppose $\left(\Phi_{1}, \Phi_{2}\right)$ are given by (3.3)-(3.4), for any $\lambda \in \mathbb{R}_{+}^{2}$ let $t \mapsto V(t, \lambda)$ be the unique positive solution to (3.8). Then we can define a transition semigroup $\left(P_{t}\right)_{t \geq 0}$ by

$$
\int_{\mathbb{M}} \mathrm{e}^{-\langle\lambda, y\rangle} P_{t}(x, \mathrm{~d} y)=\exp \{-\langle x, V(t, \lambda)\rangle\}, \quad x \in \mathbb{M} .
$$

Proof. Given $\left(\Phi_{1}, \Phi_{2}\right)$ by (3.3)-(3.4), by Proposition 3.1, there is a sequence $\left(\Phi_{k, 1}, \Phi_{k, 2}\right)$ satisfying $(\mathbf{A}, \mathbf{C}, \mathbf{D})$. By Proposition 3.2 , for any $a \geq 0$ we have $V_{k}(t, \lambda) \rightarrow V(t, \lambda)$ uniformly on $[0, a]^{3}$ 
as $k \rightarrow \infty$. Taken $x_{k} \in \mathbb{M}_{k}$ satisfying $x_{k} \rightarrow x$ as $k \rightarrow \infty$, by a continuity theorem; see, e.g., Theorem 1.18 in [21], (3.10) defines a probability measure on $\mathbb{M}$ and $\lim _{k \rightarrow \infty} P_{k}^{\left\lfloor\gamma_{k} t\right\rfloor}\left(x_{k}, \cdot\right)=P_{t}(x, \cdot)$ by weak convergence. The semigroup property of the family of $\left(P_{t}\right)_{t \geq 0}$ follows from (3.9) and (3.10).

Definition 3.1 A Markov process $\left\{Y(t)=\left(Y_{1}(t), Y_{2}(t)\right): t \geq 0\right\}$ is called a MSB-process with state space $\mathbb{M}$, if it has the transition semigroup $\left(P_{t}\right)_{t \geq 0}$ in (3.10).

Proposition 3.4 Let $\left(P_{t}\right)_{t \geq 0}$ be the transition semigroup defined by (3.10). Then we have

$$
\int_{\mathbb{M}}\langle\lambda, y\rangle P_{t}(x, \mathrm{~d} y)=\langle x, \pi(t, \lambda)\rangle, \quad \lambda \in \mathbb{R}_{+}^{2}, x \in \mathbb{M},
$$

where $t \mapsto \pi(t, \lambda)=\left(\pi_{1}(t, \lambda), \pi_{2}(t, \lambda)\right) \in \mathbb{R}_{+}^{2}$ is the unique solution to the following equations:

$$
\begin{aligned}
& \frac{\mathrm{d} \pi_{1}}{\mathrm{~d} t}(t, \lambda)=-a_{11} \pi_{1}(t, \lambda)+\pi_{2}(t, \lambda) \int_{\mathbb{M}} z_{2} n_{1}(\mathrm{~d} z), \\
& \frac{\mathrm{d} \pi_{2}}{\mathrm{~d} t}(t, \lambda)=a_{21} \pi_{1}(t, \lambda)+\int_{\mathbb{M}_{-1}}\langle z, \pi(t, \lambda)\rangle n_{2}(\mathrm{~d} z)
\end{aligned}
$$

with initial condition $\pi(0, \lambda)=\lambda$.

Proof. One can see that $V(t, 0+)=0$ for $t \geq 0$. By differentiating both sides of (3.10) with respect to $\lambda_{1}$ and $\lambda_{2}$, we have

$$
\begin{gathered}
\int_{\mathbb{M}} y_{1} P_{t}(x, \mathrm{~d} y)=x_{1} \frac{\partial V_{1}(t, 0+)}{\partial \lambda_{1}}+x_{2} \frac{\partial V_{2}(t, 0+)}{\partial \lambda_{1}}, \\
\int_{\mathbb{M}} y_{2} P_{t}(x, \mathrm{~d} y)=x_{1} \frac{\partial V_{1}(t, 0+)}{\partial \lambda_{2}}+x_{2} \frac{\partial V_{2}(t, 0+)}{\partial \lambda_{2}} .
\end{gathered}
$$

It follows from (3.8) that

$$
\begin{aligned}
& \frac{\partial V_{1}(t, 0+)}{\partial \lambda_{1}}=1+\int_{0}^{t}\left(-a_{11} \frac{\partial V_{1}(s, 0+)}{\partial \lambda_{1}}+\frac{\partial V_{2}(s, 0+)}{\partial \lambda_{1}} \int_{\mathbb{M}} z_{2} n_{1}(\mathrm{~d} z)\right) \mathrm{d} s, \\
& \frac{\partial V_{1}(t, 0+)}{\partial \lambda_{2}}=\int_{0}^{t}\left(-a_{11} \frac{\partial V_{1}(s, 0+)}{\partial \lambda_{2}}+\frac{\partial V_{2}(s, 0+)}{\partial \lambda_{2}} \int_{\mathbb{M}} z_{2} n_{1}(\mathrm{~d} z)\right) \mathrm{d} s
\end{aligned}
$$

and $\left(\bar{a}_{21}:=a_{21}+\int_{\mathbb{M}_{-1}} z_{1} n_{2}(\mathrm{~d} z)\right)$

$$
\begin{aligned}
& \frac{\partial V_{2}(t, 0+)}{\partial \lambda_{1}}=\int_{0}^{t}\left(\frac{\partial V_{1}(s, 0+)}{\partial \lambda_{1}} \bar{a}_{21}+\frac{\partial V_{2}(s, 0+)}{\partial \lambda_{1}} \int_{\mathbb{M}_{-1}} z_{2} n_{2}(\mathrm{~d} z)\right) \mathrm{d} s \\
& \frac{\partial V_{2}(t, 0+)}{\partial \lambda_{2}}=1+\int_{0}^{t}\left(\frac{\partial V_{1}(s, 0+)}{\partial \lambda_{2}} \bar{a}_{21}+\frac{\partial V_{2}(s, 0+)}{\partial \lambda_{2}} \int_{\mathbb{M}_{-1}} z_{2} n_{2}(\mathrm{~d} z)\right) \mathrm{d} s .
\end{aligned}
$$

For $\theta=\left(\theta_{1}, \theta_{2}\right) \in \mathbb{R}_{+}^{2}$ and $t \geq 0$, we define $\pi(t, \theta)=\left(\pi_{1}(t, \theta), \pi_{2}(t, \theta)\right)$ by

$$
\pi_{1}(t, \theta)=\theta_{1} \frac{\partial V_{1}(t, 0+)}{\partial \lambda_{1}}+\theta_{2} \frac{\partial V_{1}(t, 0+)}{\partial \lambda_{2}}, \quad \pi_{2}(t, \theta)=\theta_{1} \frac{\partial V_{2}(t, 0+)}{\partial \lambda_{1}}+\theta_{2} \frac{\partial V_{2}(t, 0+)}{\partial \lambda_{2}},
$$


we can conclude from the calculations above that

$$
\begin{aligned}
& \pi_{1}(t, \theta)=\theta_{1}+\int_{0}^{t}\left(-a_{11} \pi_{1}(s, \theta)+\int_{\mathbb{M}} z_{2} n_{1}(\mathrm{~d} z) \pi_{2}(s, \theta)\right) \mathrm{d} s, \\
& \pi_{2}(t, \theta)=\theta_{2}+\int_{0}^{t}\left(a_{21} \pi_{1}(s, \theta)+\int_{\mathbb{M}_{-1}}\langle z, \pi(s, \theta)\rangle n_{2}(\mathrm{~d} z)\right) \mathrm{d} s,
\end{aligned}
$$

and the desired assertion follows.

By a modification of the proof of Theorem 2.11 in [23], one can see that the semigroup defined by (3.10) is a Feller semigroup. Then the MSB-process has a càdlàg realization. Moreover, MSB-process can also be characterized in terms of a martingale problem described as follows, see Corollary 4.4 below for the proof. For $f \in C^{2}(\mathbb{M})$, let $L$ be an operator acting on $C^{2}(\mathbb{M})$ defined by

$$
\begin{gathered}
L f(x)=x_{1}\left(\alpha f_{11}^{\prime \prime}(x)-a_{11} f_{1}^{\prime}(x)+\int_{\mathbb{M}}\left\{f(x+z)-f(x)-z_{1} f_{1}^{\prime}(x)\right\} n_{1}(\mathrm{~d} z)\right) \\
+x_{2}\left(a_{21} f_{1}^{\prime}(x)+\int_{\mathbb{M}_{-1}}\{f(x+z)-f(x)\} n_{2}(\mathrm{~d} z)\right) .
\end{gathered}
$$

Suppose that $\left\{\left(Y_{1}(t), Y_{2}(t)\right): t \geq 0\right\}$ is a non-negative càdlàg process with $\mathbf{E}\left[Y_{i}(0)\right]<\infty, i=1,2$. Then $\left\{\left(Y_{1}(t), Y_{2}(t)\right): t \geq 0\right\}$ is a MSB-process with transition semigroup $\left(P_{t}\right)_{t \geq 0}$ if and only if for every $f \in C^{2}(\mathbb{M})$,

$$
f(Y(t))=f(Y(0))+\int_{0}^{t} L f(Y(s)) \mathrm{d} s+\text { local mart. }
$$

Theorem 3.1 Assume that $(\boldsymbol{A}, \boldsymbol{C}, \boldsymbol{D})$ hold, $\left(Y_{k, 1}(0) / k, Y_{k, 2}(0)\right)$ converges to $\left(Y_{1}(0), Y_{2}(0)\right)$ in distribution. Then $\left\{\left(k^{-1} Y_{k, 1}\left(\left\lfloor\gamma_{k} t\right\rfloor\right), Y_{k, 2}\left(\left\lfloor\gamma_{k} t\right\rfloor\right)\right): t \geq 0\right\}$ converges to $\left\{\left(Y_{1}(t), Y_{2}(t)\right): t \geq 0\right\}$ in distribution on $D([0, \infty), \mathbb{M})$ as $k \rightarrow \infty$.

Proof. Let $L$ be the generator of the MSB-process. For $\lambda=\left(\lambda_{1}, \lambda_{2}\right) \gg 0, x \in \mathbb{M}$, set $\mathrm{e}_{\lambda}(x)=$ $\mathrm{e}^{-\langle\lambda, x\rangle}$. We have

$$
L \mathrm{e}_{\lambda}(x)=-\mathrm{e}^{-\langle\lambda, x\rangle}\left\{x_{1} \Phi_{1}(\lambda)+x_{2} \Phi_{2}(\lambda)\right\} .
$$

Denote by $D_{1}$ the linear hull of $\left\{\mathrm{e}_{\lambda}, \lambda \gg 0\right\}$, then $D_{1}$ is an algebra which strongly separates the points of $\mathbb{M}$. Let $C_{0}(\mathbb{M})$ be the space of continuous function on $\mathbb{M}$ vanishing at infinity. By the Stone-Weierstrass theorem, $D_{1}$ is dense in $C_{0}(\mathbb{M})$ for the supremum norm. Note that $D_{1}$ is invariant under $P_{t}$ by (3.10), it follows from Proposition 3.3 in chapter I of [8] that $D_{1}$ is the core of $L$. Note that $\left\{Y_{k, 1}(n) / k, Y_{k, 2}(n): n \geq 0\right\}$ is a Markov chain with state space $\mathbb{M}_{k}$, and one-step transition probability determined by:

$$
\int_{\mathbb{M}_{k}} \mathrm{e}^{-\langle\lambda, y\rangle} P_{k}(x, \mathrm{~d} y)=\left(g_{k, 1}\left(\mathrm{e}^{-\lambda_{1} / k}, \mathrm{e}^{-\lambda_{2}}\right)\right)^{k x_{1}}\left(g_{k, 2}\left(\mathrm{e}^{-\lambda_{1} / k}, \mathrm{e}^{-\lambda_{2}}\right)\right)^{x_{2}} .
$$

The (discrete) generator $L_{k}$ of $\left\{\left(k^{-1} Y_{k, 1}\left(\left\lfloor\gamma_{k} t\right\rfloor\right), Y_{k, 2}\left(\left\lfloor\gamma_{k} t\right\rfloor\right)\right): t \geq 0\right\}$ is given by:

$$
\begin{gathered}
L_{k} \mathrm{e}_{\lambda}(x)=\gamma_{k}\left\{\left(g_{k, 1}\left(\mathrm{e}^{-\lambda_{1} / k}, \mathrm{e}^{-\lambda_{2}}\right)\right)^{k x_{1}} \cdot\left(g_{k, 2}\left(\mathrm{e}^{-\lambda_{1} / k}, \mathrm{e}^{-\lambda_{2}}\right)\right)^{x_{2}}-\mathrm{e}^{-\langle\lambda, x\rangle}\right\} \\
=\mathrm{e}^{-\langle\lambda, x\rangle} \gamma_{k}\left\{\operatorname { e x p } \left[k x_{1} \log \left(1-\left(k \gamma_{k}\right)^{-1} \Phi_{k, 1}\left(\lambda_{1}, \lambda_{2}\right) \mathrm{e}^{\lambda_{1} / k}\right)\right.\right. \\
\left.\left.+x_{2} \log \left(1-\gamma_{k}^{-1} \Phi_{k, 2}\left(\lambda_{1}, \lambda_{2}\right) \mathrm{e}^{\lambda_{2}}\right)\right]-1\right\} \\
=\mathrm{e}^{-\langle\lambda, x\rangle}\left\{x_{1} \bar{\Phi}_{k, 1}\left(\lambda_{1}, \lambda_{2}\right)+x_{2} \bar{\Phi}_{k, 2}\left(\lambda_{1}, \lambda_{2}\right)\right\}+o(1),
\end{gathered}
$$


where $\bar{\Phi}_{k, i}, \Phi_{k, i}, i=1,2$ are defined as before. It follows from Proposition 3.1 that

$$
\lim _{k \rightarrow \infty} \sup _{x \in E_{k}}\left|L_{k} \mathrm{e}_{\lambda}(x)-L \mathrm{e}_{\lambda}(x)\right|=0 .
$$

From Corollary 8.9 in Chapter 4 of $[\underline{8}$, we prove the desired result.

Theorem 3.2 Suppose that $\left\{\left(Y_{1}(t), Y_{2}(t)\right): t \geq 0\right\}$ is any MSB-process with branching mechanism $\left(\Phi_{1}, \Phi_{2}\right)$. Then there exist a sequence of positive numbers $\left\{\gamma_{k}\right\}$ and a sequence of two-type $G W$-processes $\left\{\left(Y_{k, 1}(n), Y_{k, 2}(n)\right): n \in \mathbb{N}\right\}$ with generation functions $\left(g_{k, 1}, g_{k, 2}\right)$ such that the sequence $\left\{\left(k^{-1} Y_{k, 1}\left(\left\lfloor\gamma_{k} t\right\rfloor\right), Y_{k, 2}\left(\left\lfloor\gamma_{k} t\right\rfloor\right)\right): t \geq 0\right\}$ converges in distribution on $D([0, \infty), \mathbb{M})$ to the process $\left\{\left(Y_{1}(t), Y_{2}(t)\right): t \geq 0\right\}$ as $k \rightarrow \infty$.

Proof. By Proposition 3.1, there exist $\left\{\gamma_{k}\right\},\left\{\left(g_{k, 1}, g_{k, 2}\right)\right\}$ such that conditions (A,C,D) hold. And the desired result follows from Theorem 3.1 .

\section{The construction of MSB-processes by stochastic equations}

Let $\left(\Omega, \mathcal{F}, \mathcal{F}_{t}, \mathbf{P}\right)$ be a complete filtered probability space satisfying the usual hypotheses, let $\{B(t)\}$ be a standard Brownian motion, $\left\{N_{1}(\mathrm{~d} s, \mathrm{~d} u, \mathrm{~d} z)\right\}$ be a Poisson random measure on $(0, \infty)^{2} \times \mathbb{M}$ with intensity $\mathrm{d} s \mathrm{~d} u n_{1}(\mathrm{~d} z),\left\{N_{2}(\mathrm{~d} s, \mathrm{~d} u, \mathrm{~d} z)\right\}$ be a Poisson random measure on $(0, \infty)^{2} \times \mathbb{M}_{-1}$ with intensity $\mathrm{d} s \mathrm{~d} u n_{2}(\mathrm{~d} z), z=\left(z_{1}, z_{2}\right)$. Suppose that $B, N_{1}, N_{2}$ are independent of each other. Let us recall the stochastic integral equation system (1.7)-(1.8):

$$
\begin{aligned}
Y_{1}(t)=Y_{1}(0) & -\int_{0}^{t} a_{11} Y_{1}(s) \mathrm{d} s+\int_{0}^{t} \sqrt{2 \alpha Y_{1}(s)} \mathrm{d} B(s)+\int_{0}^{t} \int_{0}^{Y_{1}(s-)} \int_{\mathbb{M}} z_{1} \tilde{N}_{1}(\mathrm{~d} s, \mathrm{~d} u, \mathrm{~d} z) \\
& +\int_{0}^{t} a_{21} Y_{2}(s) \mathrm{d} s+\int_{0}^{t} \int_{0}^{Y_{2}(s-)} \int_{\mathbb{M}_{-1}} z_{1} N_{2}(\mathrm{~d} s, \mathrm{~d} u, \mathrm{~d} z), \\
Y_{2}(t)=Y_{2}(0) & +\int_{0}^{t} \int_{0}^{Y_{1}(s-)} \int_{\mathbb{M}} z_{2} N_{1}(\mathrm{~d} s, \mathrm{~d} u, \mathrm{~d} z)+\int_{0}^{t} \int_{0}^{Y_{2}(s-)} \int_{\mathbb{M}_{-1}} z_{2} N_{2}(\mathrm{~d} s, \mathrm{~d} u, \mathrm{~d} z),
\end{aligned}
$$

where $\tilde{N}_{1}(\mathrm{~d} s, \mathrm{~d} u, \mathrm{~d} z)=N_{1}(\mathrm{~d} s, \mathrm{~d} u, \mathrm{~d} z)-\mathrm{d} s \mathrm{~d} u n_{1}(\mathrm{~d} z)$ is the compensated Poisson random measure of $N_{1}$.

Proposition 4.1 Suppose that $\{Y(t)\}$ satisfies (1.7)-(1.8) and $\mathbf{P}\{Y(0) \geq \mathbf{0}\}=1$. Then $\mathbf{P}\{Y(t) \geq \mathbf{0}, \forall t \geq 0\}=1$.

Proof. By equation (1.8), if $Y_{2}(0) \geq 0$, it is not hard to see that for all $t \geq 0, Y_{2}(t) \geq 0$. Now suppose that there exists $\varepsilon>0$, such that $\tau:=\inf \left\{t>0, Y_{1}(t) \leq-\varepsilon\right\}<\infty$ with strictly positive probability. Then there exists $t_{0}>0, Y_{1}\left(t_{0}\right)=0$, and on the time interval $\left[t_{0}, \tau\right], t \mapsto Y_{1}(t)$ is a strictly negative continuous function. Hence there are some $t_{1} \in\left[t_{0}, \tau\right]$ and $\delta>0$, such that for all $s \in\left[t_{0}, t_{1}\right],-a_{11} Y_{1}(s)+a_{21} Y_{2}(s) \geq \delta$. Then

$$
Y_{1}\left(t_{1}\right)=Y_{1}\left(t_{1}\right)-Y_{1}\left(t_{0}\right) \geq \int_{t_{0}}^{t_{1}}\left(-a_{11} Y_{1}(s)+a_{21} Y_{2}(s)\right) \mathrm{d} s \geq \delta\left(t_{1}-t_{0}\right)>0,
$$


since $Y_{1}(t)<0, \forall t \in\left(t_{0}, \tau\right]$, we get a contradiction.

To analyze the property of above equation system, we first construct a sequence of functions $\left\{\phi_{k}\right\}$ on $\mathbb{R}$ as follows. For each integer $k \geq 0$ define $a_{k}=\exp \{-k(k+1) / 2\}$. Then $a_{k} \rightarrow 0$ decreasingly as $k \rightarrow \infty$ and $\int_{a_{k}}^{a_{k-1}} z^{-1} \mathrm{~d} z=k$ for $k \geq 1$. Let $x \mapsto \psi_{k}(x)$ be a non-negative continuous function on $\mathbb{R}$ which has support in $\left(a_{k}, a_{k-1}\right)$ and satisfies $\int_{a_{k}}^{a_{k-1}} \psi_{k}(x) \mathrm{d} x=1$ and $0 \leq \psi_{k}(x) \leq 2(k x)^{-1}$ for $a_{k}<x<a_{k-1}$. For each $k \geq 1$ let

$$
\phi_{k}(z)=\int_{0}^{|z|} \mathrm{d} y \int_{0}^{y} \psi_{k}(x) \mathrm{d} x, \quad z \in \mathbb{R} .
$$

Moreover, for function $f$ on $\mathbb{R}$ we denote

$$
\Delta_{z} f(x)=f(x+z)-f(x) .
$$

Theorem 4.1 The pathwise uniqueness for (1.7)-(1.8) holds.

Proof. Suppose that $\{Y(t)\}$ and $\left\{Y^{\prime}(t)\right\}$ are two solutions of (1.7)-(1.8). Let $\zeta_{i}(t)=Y_{i}(t)-$ $Y_{i}^{\prime}(t), i=1,2$ for $t \geq 0$. We have

$$
\begin{aligned}
\zeta_{1}(t)=\zeta_{1}(0) & -\int_{0}^{t}\left(a_{11} \zeta_{1}(s)+a_{21} \zeta_{2}(s)\right) \mathrm{d} s+\int_{0}^{t}\left(\sqrt{2 \alpha Y_{1}(s)}-\sqrt{2 \alpha Y_{1}^{\prime}(s)}\right) \mathrm{d} B(s) \\
& +\int_{0}^{t} \int_{Y_{1}^{\prime}(s-)}^{Y_{1}(s-)} \int_{\mathbb{M}^{\prime}} z_{1} \mathbf{1}_{\left\{\zeta_{1}(s-)>0\right\}} \tilde{N}_{1}(\mathrm{~d} s, \mathrm{~d} u, \mathrm{~d} z) \\
& -\int_{0}^{t} \int_{Y_{1}(s-)}^{Y_{1}^{\prime}(s-)} \int_{\mathbb{M}_{1}} z_{1} \mathbf{1}_{\left\{\zeta_{1}(s-) \leq 0\right\}} \tilde{N}_{1}(\mathrm{~d} s, \mathrm{~d} u, \mathrm{~d} z) \\
& +\int_{0}^{t} \int_{Y_{2}^{\prime}(s-)}^{Y_{2}(s-)} \int_{\mathbb{M}_{-1}} z_{1} \mathbf{1}_{\left\{\zeta_{2}(s-)>0\right\}} N_{2}(\mathrm{~d} s, \mathrm{~d} u, \mathrm{~d} z) \\
& -\int_{0}^{t} \int_{Y_{2}(s-)}^{Y_{2}^{\prime}(s-)} \int_{\mathbb{M}_{-1}} z_{1} \mathbf{1}_{\left\{\zeta_{2}(s-) \leq 0\right\}} N_{2}(\mathrm{~d} s, \mathrm{~d} u, \mathrm{~d} z)
\end{aligned}
$$

Let $\tau_{m}=\inf \left\{t \geq 0: Y_{1}(t) \vee Y_{2}(t) \vee Y_{1}^{\prime}(t) \vee Y_{2}^{\prime}(t) \geq m\right\}$ for $m \geq 1$. By similar calculations as in Theorem 3.4 in [27], there exists $C_{1}>0$ such that

$$
\mathbf{E}\left|\zeta_{1}\left(t \wedge \tau_{m}\right)\right| \leq C_{1} \int_{0}^{t} \mathbf{E}\left(\left|\zeta_{1}\left(s \wedge \tau_{m}\right)\right|+\left|\zeta_{2}\left(s \wedge \tau_{m}\right)\right|\right) \mathrm{d} s
$$

On the other hand,

$$
\begin{aligned}
\zeta_{2}(t)=\zeta_{2}(0) & +\int_{0}^{t} \int_{Y_{1}^{\prime}(s-)}^{Y_{1}(s-)} \int_{\mathbb{M}} z_{2} \mathbf{1}_{\left\{\zeta_{1}(s-)>0\right\}} N_{1}(\mathrm{~d} s, \mathrm{~d} u, \mathrm{~d} z) \\
& -\int_{0}^{t} \int_{Y_{1}(s-)}^{Y_{1}^{\prime}(s-)} \int_{\mathbb{M}_{2}} z_{2} \mathbf{1}_{\left\{\zeta_{1}(s-) \leq 0\right\}} N_{1}(\mathrm{~d} s, \mathrm{~d} u, \mathrm{~d} z) \\
& +\int_{0}^{t} \int_{Y_{2}^{\prime}(s-)}^{Y_{2}(s-)} \int_{\mathbb{M}_{-1}} z_{2} \mathbf{1}_{\left\{\zeta_{2}(s-)>0\right\}} N_{2}(\mathrm{~d} s, \mathrm{~d} u, \mathrm{~d} z) \\
& -\int_{0}^{t} \int_{Y_{2}(s-)}^{Y_{2}^{\prime}(s-)} \int_{\mathbb{M}_{-1}} z_{2} \mathbf{1}_{\left\{\zeta_{2}(s-) \leq 0\right\}} N_{2}(\mathrm{~d} s, \mathrm{~d} u, \mathrm{~d} z) .
\end{aligned}
$$


By Itô's formula,

$$
\begin{aligned}
\phi_{k}\left(\zeta_{2}\left(t \wedge \tau_{m}\right)\right)= & \phi_{k}\left(\zeta_{2}(0)\right)+\int_{0}^{t \wedge \tau_{m}} \int_{Y_{1}^{\prime}(s-)}^{Y_{1}(s-)} \int_{\mathbb{M}} \Delta_{z_{2}} \phi_{k}\left(\zeta_{2}(s-)\right) \mathbf{1}_{\left\{\zeta_{1}(s-)>0\right\}} \mathrm{d} s \mathrm{~d} u n_{1}(\mathrm{~d} z) \\
& +\int_{0}^{t \wedge \tau_{m}} \int_{Y_{1}(s-)}^{Y_{1}^{\prime}(s-)} \int_{\mathbb{M}} \Delta_{-z_{2}} \phi_{k}\left(\zeta_{2}(s-)\right) \mathbf{1}_{\left\{\zeta_{1}(s-) \leq 0\right\}} \mathrm{d} s \mathrm{~d} u n_{1}(\mathrm{~d} z) \\
& +\int_{0}^{t \wedge \tau_{m}} \int_{Y_{2}^{\prime}(s-)}^{Y_{2}(s-)} \int_{\mathbb{M}_{-1}} \Delta_{z_{2}} \phi_{k}\left(\zeta_{2}(s-)\right) \mathbf{1}_{\left\{\zeta_{2}(s-)>0\right\}} \mathrm{d} s \mathrm{~d} u n_{2}(\mathrm{~d} z) \\
& +\int_{0}^{t \wedge \tau_{m}} \int_{Y_{2}(s-)}^{Y_{2}^{\prime}(s-)} \int_{\mathbb{M}_{-1}} \Delta_{-z_{2}} \phi_{k}\left(\zeta_{2}(s-)\right) \mathbf{1}_{\left\{\zeta_{2}(s-) \leq 0\right\}} \mathrm{d} s \mathrm{~d} u n_{2}(\mathrm{~d} z)+\text { mart. }
\end{aligned}
$$

Similarly, there exists $C_{2}>0$ such that

$$
\mathbf{E}\left|\zeta_{2}\left(t \wedge \tau_{m}\right)\right| \leq C_{2} \int_{0}^{t} \mathbf{E}\left(\left|\zeta_{1}\left(s \wedge \tau_{m}\right)\right|+\left|\zeta_{2}\left(s \wedge \tau_{m}\right)\right|\right) \mathrm{d} s
$$

In conclusion,

$$
\mathbf{E}\left(\left|\zeta_{1}\left(t \wedge \tau_{m}\right)\right|+\left|\zeta_{2}\left(t \wedge \tau_{m}\right)\right|\right) \leq\left(C_{1}+C_{2}\right) \int_{0}^{t} \mathbf{E}\left(\left|\zeta_{1}\left(s \wedge \tau_{m}\right)\right|+\left|\zeta_{2}\left(s \wedge \tau_{m}\right)\right|\right) \mathrm{d} s .
$$

By Gronwall inequality, for all $t \geq 0$,

$$
\mathbf{E}\left(\left|\zeta_{1}\left(t \wedge \tau_{m}\right)\right|+\left|\zeta_{2}\left(t \wedge \tau_{m}\right)\right|\right)=0
$$

Since $\{Y(t)\}$ and $\left\{Y^{\prime}(t)\right\}$ have càdlàg sample paths, we conclude that $\mathbf{P}\left\{Y(t)=Y^{\prime}(t), \forall t \geq\right.$ $0\}=1$ as $m \rightarrow \infty$.

Theorem 4.2 There is a unique non-negative strong solution to (1.7)-(1.8).

Proof. Since $\nu_{1}$ is supported on $\mathbb{M} \backslash\{\mathbf{0}\}$, we can rewrite (1.7)-(1.8) as

$$
\begin{aligned}
Y_{1}(t)=Y_{1}(0) & +\int_{0}^{t}\left(a_{21} Y_{2}(s)-a_{11} Y_{1}(s)\right) \mathrm{d} s+\int_{0}^{t} \sqrt{2 \alpha Y_{1}(s)} \mathrm{d} B(s) \\
& +\int_{0}^{t} \int_{0}^{Y_{1}(s-)} \int_{\mathbb{M}_{-1}} z_{1} \tilde{N}_{1}(\mathrm{~d} s, \mathrm{~d} u, \mathrm{~d} z)+\int_{0}^{t} \int_{0}^{Y_{2}(s-)} \int_{\mathbb{M}_{-1}} z_{1} N_{2}(\mathrm{~d} s, \mathrm{~d} u, \mathrm{~d} z), \\
Y_{2}(t)=Y_{2}(0) & +\int_{0}^{t} \int_{0}^{Y_{1}(s-)} \int_{\mathbb{M}_{-1}} z_{2} N_{1}(\mathrm{~d} s, \mathrm{~d} u, \mathrm{~d} z)+\int_{0}^{t} \int_{0}^{Y_{2}(s-)} \int_{\mathbb{M}_{-1}} z_{2} N_{2}(\mathrm{~d} s, \mathrm{~d} u, \mathrm{~d} z) .
\end{aligned}
$$

For any fixed $n \geq 1$, let $V_{n}=\left\{z \in \mathbb{M}_{-1}:\|z\| \geq 1 / n\right\}$, then $n_{1}\left(V_{n}\right)+n_{2}\left(V_{n}\right)<\infty$. For $m \geq 1$ and $x \in \mathbb{M}$, define

$$
\begin{aligned}
& b(x, m)=a_{21}\left(x_{2} \wedge m\right)-a_{11}\left(x_{1} \wedge m\right), \quad \theta(m, n)=\int_{V_{n}}\left(z_{1} \wedge m\right) n_{1}(\mathrm{~d} z), \\
& \beta_{1}(m)=\int_{\mathbb{M}_{-1}}\left(z_{1}-z_{1} \wedge m\right) n_{1}(\mathrm{~d} z), \quad \beta_{2}(m)=\int_{\mathbb{M}_{-1}}\left(z_{2}-z_{2} \wedge m\right) n_{2}(\mathrm{~d} z) .
\end{aligned}
$$


By the results for continuous-type stochastic equations in [15, p.169], one can show that there is a non-negative weak solution to the following stochastic equation system:

$$
\begin{aligned}
Y_{1}(t)=Y_{1}(0) & +\int_{0}^{t}\left(b(Y(s), m)-\left(\beta_{1}(m)+\theta(m, n)\right)\left(Y_{1}(s) \wedge m\right)\right) \mathrm{d} s \\
& +\int_{0}^{t} \sqrt{2 \alpha\left(Y_{1}(s) \wedge m\right)} \mathrm{d} B(s) \\
Y_{2}(t)=Y_{2}(0) & -\int_{0}^{t} \beta_{2}(m)\left(Y_{2}(s) \wedge m\right) \mathrm{d} s .
\end{aligned}
$$

The pathwise uniqueness holds for the above system of equations by similar arguments as in Theorem 4.1. Then it has a unique strong solution. By similar arguments as in the proof of Proposition 2.2 in [13], we can get a pathwise unique non-negative strong solution $\left\{Y_{m, n}(t): t \geq\right.$ $0\}$ to (4.1)-(4.2):

$$
\begin{aligned}
Y_{1}(t)=Y_{1}(0) & +\int_{0}^{t} b(Y(s), m)-\beta_{1}(m)\left(Y_{1}(s) \wedge m\right) \mathrm{d} s+\int_{0}^{t} \sqrt{2 \alpha\left(Y_{1}(s) \wedge m\right)} \mathrm{d} B(s) \\
& +\int_{0}^{t} \int_{0}^{Y_{1}(s-) \wedge m} \int_{V_{n}}\left(z_{1} \wedge m\right) \tilde{N}_{1}(\mathrm{~d} s, \mathrm{~d} u, \mathrm{~d} z) \\
& +\int_{0}^{t} \int_{0}^{Y_{2}(s-) \wedge m} \int_{V_{n}}\left(z_{1} \wedge m\right) N_{2}(\mathrm{~d} s, \mathrm{~d} u, \mathrm{~d} z), \\
Y_{2}(t)=Y_{2}(0) & -\int_{0}^{t} \beta_{2}(m)\left(Y_{2}(s) \wedge m\right) \mathrm{d} s+\int_{0}^{t} \int_{0}^{Y_{1}(s-) \wedge m} \int_{V_{n}}\left(z_{2} \wedge m\right) N_{1}(\mathrm{~d} s, \mathrm{~d} u, \mathrm{~d} z) \\
& +\int_{0}^{t} \int_{0}^{Y_{2}(s-) \wedge m} \int_{V_{n}}\left(z_{2} \wedge m\right) N_{2}(\mathrm{~d} s, \mathrm{~d} u, \mathrm{~d} z) .
\end{aligned}
$$

As in the proof of Lemma 4.3 in [13], one can see the sequence $\left\{Y_{m, n}(t): t \geq 0\right\}, n=1,2, \ldots$ is tight in $D([0, \infty), \mathbb{M})$. Following the proof of Theorem 4.4 in [13], it is easy to show that any weak limit point $\left\{Y_{m}(t): t \geq 0\right\}$ of the sequence is a non-negative weak solution to

$$
\begin{aligned}
Y_{1}(t)=Y_{1}(0) & +\int_{0}^{t} b(Y(s), m)-\beta_{1}(m)\left(Y_{1}(s) \wedge m\right) \mathrm{d} s+\int_{0}^{t} \sqrt{2 \alpha\left(Y_{1}(s) \wedge m\right)} \mathrm{d} B(s) \\
& +\int_{0}^{t} \int_{0}^{Y_{1}(s-) \wedge m} \int_{\mathbb{M}_{-1}}\left(z_{1} \wedge m\right) \tilde{N}_{1}(\mathrm{~d} s, \mathrm{~d} u, \mathrm{~d} z) \\
& +\int_{0}^{t} \int_{0}^{Y_{2}(s-) \wedge m} \int_{\mathbb{M}_{-1}}\left(z_{1} \wedge m\right) N_{2}(\mathrm{~d} s, \mathrm{~d} u, \mathrm{~d} z), \\
Y_{2}(t)=Y_{2}(0) & -\int_{0}^{t} \beta_{2}(m)\left(Y_{2}(s) \wedge m\right) \mathrm{d} s+\int_{0}^{t} \int_{0}^{Y_{1}(s-) \wedge m} \int_{\mathbb{M}_{-1}}\left(z_{2} \wedge m\right) N_{1}(\mathrm{~d} s, \mathrm{~d} u, \mathrm{~d} z) \\
& +\int_{0}^{t} \int_{0}^{Y_{2}(s-) \wedge m} \int_{\mathbb{M}_{-1}}\left(z_{2} \wedge m\right) N_{2}(\mathrm{~d} s, \mathrm{~d} u, \mathrm{~d} z) .
\end{aligned}
$$

By Theorem 4.1, the pathwise uniqueness holds for (4.3)-(4.4), so the system of equations has a unique strong solution. Finally, the desired result follows from a modification of the proof of Proposition 2.4 in [13]. 
Corollary 4.1 A càdlàg non-negative process is a MSB-process with transition semigroup $\left(P_{t}\right)_{t \geq 0}$ defined by (3.8) and (3.10) if and only if it is a weak solution of (1.7)-(1.8).

Proof. Suppose that $\left\{\left(Y_{1}(t), Y_{2}(t)\right)\right\}_{t \geq 0}$ is a weak solution of (1.7)-(1.8). By Itô's formula one can see that $\left\{\left(Y_{1}(t), Y_{2}(t)\right)\right\}_{t \geq 0}$ solves the martingale problem associated with the generator $L$. By the arguments in Section 3 we infer that $\left\{\left(Y_{1}(t), Y_{2}(t)\right)\right\}_{t \geq 0}$ is a MSB-process with transition semigroup $\left(P_{t}\right)_{t \geq 0}$ defined by (3.8) and (3.10). Conversely, suppose that $\left\{\left(Y_{1}(t), Y_{2}(t)\right)\right\}_{t \geq 0}$ is a càdlàg realization of MSB-process with transition semigroup $\left(P_{t}\right)_{t \geq 0}$ defined by (3.8) and (3.10). Then the distributions of $\left\{\left(Y_{1}(t), Y_{2}(t)\right)\right\}_{t \geq 0}$ on $D([0, \infty), \mathbb{M})$ can be characterized uniquely by the martingale problem. By a standard stopping time argument, we have

$$
\begin{aligned}
& Y_{1}(t)=Y_{1}(0)-\int_{0}^{t}\left(a_{11} Y_{1}(s)-a_{21} Y_{2}(s)\right) \mathrm{d} s+\int_{0}^{t} \int_{\mathbb{M}_{-1}} Y_{2}(s) z_{1} \mathrm{~d} s n_{2}(\mathrm{~d} z)+G_{1}(t), \\
& Y_{2}(t)=Y_{2}(0)+\int_{0}^{t} \int_{\mathbb{M}} Y_{1}(s) z_{2} \mathrm{~d} s n_{1}(\mathrm{~d} z)+\int_{0}^{t} \int_{\mathbb{M}_{-1}} Y_{2}(s) z_{2} \mathrm{~d} s n_{2}(\mathrm{~d} z)+G_{2}(t),
\end{aligned}
$$

where $G_{1}(t)$ and $G_{2}(t)$ are two square-integrable local martingales. Let $N_{0}(\mathrm{~d} s, \mathrm{~d} z)$ be the optimal random measure on $[0, \infty) \times \mathbb{M}_{-1}$ defined by

$$
N_{0}(\mathrm{~d} s, \mathrm{~d} z):=\sum_{s>0} \mathbf{1}_{\left\{\left(Y_{1}(s), Y_{2}(s)\right) \neq\left(Y_{1}(s-), Y_{2}(s-)\right)\right\}} \delta_{(s, Y(s)-Y(s-))}(\mathrm{d} s, \mathrm{~d} z),
$$

It follows from [7, p.376] that

$$
G_{1}(t)=G_{1}^{c}(t)+\int_{0}^{t} \int_{\mathbb{M}_{-1}} z_{1} \tilde{N}_{0}(\mathrm{~d} s, \mathrm{~d} z), \quad G_{2}(t)=G_{2}^{c}(t)+\int_{0}^{t} \int_{\mathbb{M}_{-1}} z_{2} \tilde{N}_{0}(\mathrm{~d} s, \mathrm{~d} z),
$$

where $t \mapsto G_{1}^{c}(t)$ and $t \mapsto G_{2}^{c}(t)$ are two continuous local martingales with quadratic variations $t \mapsto C_{1}(t)$ and $t \mapsto C_{2}(t)$, respectively. Using Itô's formula to (4.5)-(4.6) and the uniqueness of canonical decompositions of semi-martingales we find that $N_{0}(\mathrm{~d} s, \mathrm{~d} z)$ has a predictable compensator

$$
\hat{N}_{0}(\mathrm{~d} s, \mathrm{~d} z)=Y_{1}(s-) \mathrm{d} s n_{1}(\mathrm{~d} z)+Y_{2}(s-) \mathrm{d} s n_{2}(\mathrm{~d} z),
$$

$\mathrm{d} C_{1}(t)=2 \alpha Y_{1}(t) \mathrm{d} t$ and $\mathrm{d} C_{2}(t)=0$. Then we obtain the equation (1.7)-(1.8) on an extension of the probability space by applying martingale representation theorems; see, e.g., [15, p.93, p.84], which completes the proof.

\section{The distribution of local jumps}

For any initial time $r \geq 0$, let $Y=\left(\Omega, \mathcal{F}, \mathcal{F}_{r, t}, Y(t), \mathbf{P}_{r, y}: t \geq r, y \geq 0\right)$ be a Hunt realization of the MSB-process with transition semigroup $\left(P_{t}\right)_{t \geq 0}$ defined by (3.8) and (3.10). Here, $\left\{\mathbf{P}_{r, y}\right.$ : $y \geq 0\}$ be a family of probability measures on $\left(\Omega, \mathcal{F}, \mathcal{F}_{r, t}\right)$ satisfying $\mathbf{P}_{r, y}\{Y(r)=y\}=1$ for all $y \geq 0$. For any $t \geq r \geq 0$ and $\lambda \in[0, \infty)^{2}$, we have

$$
\mathbf{P}_{r, y} \exp \{-\langle\lambda, Y(t)\rangle\}=\exp \{-\langle y, \bar{V}(r, \lambda)\rangle\},
$$


where $r \rightarrow \bar{V}(r, \lambda):=V(t-r, \lambda)$ satisfies

$$
\bar{V}_{1}(r, \lambda)=\int_{r}^{t} \Phi_{1}(\bar{V}(s, \lambda)) \mathrm{d} s+\lambda_{1}, \quad \bar{V}_{2}(r, \lambda)=\int_{r}^{t} \Phi_{2}(\bar{V}(s, \lambda)) \mathrm{d} s+\lambda_{2}, \quad 0 \leq r \leq t .
$$

By modifying the arguments of Proposition 4.1, Theorem 4.2 and Corollary 4.4 in [23], we have the following

Proposition 5.1 For $\left\{t_{1}<\ldots<t_{n}\right\} \subset[0, \infty)$ and $\left\{\lambda_{1}, \ldots \lambda_{n}\right\} \subset[0, \infty)^{2}$, we have

$$
\mathbf{P}_{r, y} \exp \left\{-\sum_{j=1}^{n}\left\langle\lambda_{j}, Y\left(t_{j}\right) \mathbf{1}_{\left\{r \leq t_{j}\right\}}\right\rangle\right\}=\exp \{-\langle y, \bar{V}(r)\rangle\}, \quad 0 \leq r \leq t_{n},
$$

where $\bar{V}(r)=\bar{V}\left(r, \lambda_{1}, \ldots, \lambda_{n}\right)$ on $\left[0, t_{n}\right]$ satisfies

$$
\bar{V}_{1}(r)=\int_{r}^{t_{n}} \Phi_{1}(\bar{V}(s)) \mathrm{d} s+\sum_{j=1}^{n} \lambda_{j 1} \mathbf{1}_{\left\{r \leq t_{j}\right\}}, \quad \bar{V}_{2}(r)=\int_{r}^{t_{n}} \Phi_{2}(\bar{V}(s)) \mathrm{d} s+\sum_{j=1}^{n} \lambda_{j 2} \mathbf{1}_{\left\{r \leq t_{j}\right\}} .
$$

Proposition 5.2 Suppose that $t \geq 0$ and $\mu$ is a finite measure supported by $[0, t]$. Let $s \mapsto$ $\lambda(s)=\left(\lambda_{1}(s), \lambda_{2}(s)\right)$ be a bounded positive Borel function on $[0, t]$, then we have

$$
\mathbf{P}_{r, y} \exp \left\{-\int_{[r, t]}\langle\lambda(s), Y(s)\rangle \mu(\mathrm{d} s)\right\}=\exp \{-\langle y, \bar{V}(r)\rangle\}, \quad 0 \leq r \leq t,
$$

where $r \mapsto \bar{V}(r)=\bar{V}\left(r, \lambda_{1}, \lambda_{2}\right)$ is the positive solution on $[0, t]$ of

$$
\bar{V}_{1}(r)=\int_{r}^{t} \Phi_{1}(\bar{V}(s)) \mathrm{d} s+\int_{r}^{t} \lambda_{1}(s) \mu(\mathrm{d} s), \quad \bar{V}_{2}(r)=\int_{r}^{t} \Phi_{2}(\bar{V}(s)) \mathrm{d} s+\int_{r}^{t} \lambda_{2}(s) \mu(\mathrm{d} s) .
$$

Corollary 5.1 Let $Y=\left(\Omega, \mathcal{F}, \mathcal{F}_{t}, Y(t), \mathbf{P}_{y}\right)$ be a Hunt realization of the MSB-process started from time zero. Then for $t \geq 0, \lambda=\left(\lambda_{1}, \lambda_{2}\right) \in[0, \infty)^{2}$, we have

$$
\mathbf{P}_{y} \exp \left\{-\int_{0}^{t}\langle\lambda, Y(s)\rangle \mathrm{d} s\right\}=\exp \{-\langle y, \bar{V}(t)\rangle\}
$$

where $t \mapsto \bar{V}(t)=\bar{V}(t, \lambda)$ is the positive solution of

$$
\bar{V}_{1}(t)=\int_{0}^{t} \Phi_{1}(\bar{V}(s)) \mathrm{d} s+\lambda_{1} t, \quad \bar{V}_{2}(t)=\int_{0}^{t} \Phi_{2}(\bar{V}(s)) \mathrm{d} s+\lambda_{2} t .
$$

We shall introduce some notations before presenting the main results in this section. Let $r=\left(r_{1}, r_{2}\right) \in[0, \infty)^{2}, A_{r}=\left(r_{1}, \infty\right) \times\left(r_{2}, \infty\right)$ and $n\left(A_{r}\right)=\left(n_{1}\left(A_{r}\right), n_{2}\left(A_{r}\right)\right)$. We define two functions on $[0, \infty)^{2}$ :

$$
\begin{aligned}
& \Phi_{1}^{r}\left(\lambda_{1}, \lambda_{2}\right)=-a_{11}^{r} \lambda_{1}+b_{11}^{r} \lambda_{2}-\alpha \lambda_{1}^{2}-\int_{\mathbb{M} \backslash A_{r}}\left(\mathrm{e}^{-\langle\lambda, z\rangle}-1+\langle\lambda, z\rangle\right) n_{1}(\mathrm{~d} z), \\
& \Phi_{2}^{r}\left(\lambda_{1}, \lambda_{2}\right)=a_{21}^{r} \lambda_{1}+b_{21}^{r} \lambda_{2}-\int_{\mathbb{M}_{-1} \backslash A_{r}}\left(\mathrm{e}^{-\langle\lambda, z\rangle}-1+\langle\lambda, z\rangle\right) n_{2}(\mathrm{~d} z),
\end{aligned}
$$


where

$$
\begin{aligned}
& a_{11}^{r}=a_{11}+\int_{A_{r}} z_{1} n_{1}(\mathrm{~d} z), \quad a_{21}^{r}=a_{21}+\int_{\mathbb{M}_{-1} \backslash A_{r}} z_{1} n_{2}(\mathrm{~d} z), \\
& b_{11}^{r}=\int_{\mathbb{M} \backslash A_{r}} z_{2} n_{1}(\mathrm{~d} z), \quad b_{21}^{r}=\int_{\mathbb{M}_{-1} \backslash A_{r}} z_{2} n_{2}(\mathrm{~d} z) .
\end{aligned}
$$

The following theorem gives a characterization of the distribution of the local maximal jump of the MSB-process.

Theorem 5.1 Let $\tau_{r}=\inf \left\{s \geq 0: \Delta Y_{1}(s)>r_{1}\right.$ or $\left.\Delta Y_{2}(s)>r_{2}\right\}$. Then we have

$$
\mathbf{P}_{y}\left(\tau_{r}>t\right)=\exp \left\{-\left\langle y, \bar{V}^{r}\left(t, n\left(A_{r}\right)\right)\right\rangle\right\}
$$

where $\bar{V}^{r}(t)=\left(\bar{V}_{1}^{r}(t), \bar{V}_{2}^{r}(t)\right)$ is the solution of

$$
\bar{V}_{1}^{r}(t)=\int_{0}^{t} \Phi_{1}^{r}\left(\bar{V}^{r}(s)\right) \mathrm{d} s+n_{1}\left(A_{r}\right) t, \quad \bar{V}_{2}^{r}(t)=\int_{0}^{t} \Phi_{2}^{r}\left(\bar{V}^{r}(s)\right) \mathrm{d} s+n_{2}\left(A_{r}\right) t .
$$

Proof. We can rewrite equations (1.7)-(1.8) by:

$$
\begin{aligned}
Y_{1}(t) & =Y_{1}(0)+\int_{0}^{t}\left(a_{21}^{r} Y_{2}(s)-a_{11}^{r} Y_{1}(s)\right) \mathrm{d} s+\int_{0}^{t} \sqrt{2 \alpha Y_{1}(s)} \mathrm{d} B(s) \\
& +\int_{0}^{t} \int_{0}^{Y_{1}(s-)} \int_{\mathbb{M} \backslash A_{r}} z_{1} \tilde{N}_{1}(\mathrm{~d} s, \mathrm{~d} u, \mathrm{~d} z)+\int_{0}^{t} \int_{0}^{Y_{2}(s-)} \int_{\mathbb{M}_{-1} \backslash A_{r}} z_{1} \tilde{N}_{2}(\mathrm{~d} s, \mathrm{~d} u, \mathrm{~d} z) \\
& +\int_{0}^{t} \int_{0}^{Y_{1}(s-)} \int_{A_{r}} z_{1} N_{1}(\mathrm{~d} s, \mathrm{~d} u, \mathrm{~d} z)+\int_{0}^{t} \int_{0}^{Y_{2}(s-)} \int_{A_{r}} z_{1} N_{2}(\mathrm{~d} s, \mathrm{~d} u, \mathrm{~d} z), \\
Y_{2}(t) & =Y_{2}(0)+\int_{0}^{t}\left(b_{11}^{r} Y_{1}(s)+b_{21}^{r} Y_{2}(s)\right) \mathrm{d} s \\
& +\int_{0}^{t} \int_{0}^{Y_{1}(s-)} \int_{\mathbb{M} \backslash A_{r}} z_{2} \tilde{N}_{1}(\mathrm{~d} s, \mathrm{~d} u, \mathrm{~d} z)+\int_{0}^{t} \int_{0}^{Y_{2}(s-)} \int_{\mathbb{M}_{-1} \backslash A_{r}} z_{2} \tilde{N}_{2}(\mathrm{~d} s, \mathrm{~d} u, \mathrm{~d} z) \\
& +\int_{0}^{t} \int_{0}^{Y_{1}(s-)} \int_{A_{r}} z_{2} N_{1}(\mathrm{~d} s, \mathrm{~d} u, \mathrm{~d} z)+\int_{0}^{t} \int_{0}^{Y_{2}(s-)} \int_{A_{r}} z_{2} N_{2}(\mathrm{~d} s, \mathrm{~d} u, \mathrm{~d} z) .
\end{aligned}
$$

Let $\left(\left(Y_{1}^{r_{1}}(t), Y_{2}^{r_{2}}(t)\right): t \geq 0\right)$ be the strong solution to

$$
\begin{aligned}
Y_{1}^{r_{1}}(t) & =Y_{1}(0)+\int_{0}^{t}\left(a_{21}^{r} Y_{2}^{r_{2}}(s)-a_{11}^{r} Y_{1}^{r_{1}}(s)\right) \mathrm{d} s+\int_{0}^{t} \sqrt{2 \alpha Y_{1}^{r_{1}}(s)} \mathrm{d} B(s) \\
& +\int_{0}^{t} \int_{0}^{Y_{1}^{r_{1}}(s-)} \int_{\mathbb{M} \backslash A_{r}} z_{1} \tilde{N}_{1}(\mathrm{~d} s, \mathrm{~d} u, \mathrm{~d} z)+\int_{0}^{t} \int_{0}^{Y_{2}^{r_{2}}(s-)} \int_{\mathbb{M}_{-1} \backslash A_{r}} z_{1} \tilde{N}_{2}(\mathrm{~d} s, \mathrm{~d} u, \mathrm{~d} z), \\
Y_{2}^{r_{2}}(t) & =Y_{2}(0)+\int_{0}^{t}\left(b_{11}^{r} Y_{1}^{r_{1}}(s)+b_{21}^{r} Y_{2}^{r_{2}}(s)\right) \mathrm{d} s \\
& +\int_{0}^{t} \int_{0}^{Y_{1}^{r_{1}}(s-)} \int_{\mathbb{M} \backslash A_{r}} z_{2} \tilde{N}_{1}(\mathrm{~d} s, \mathrm{~d} u, \mathrm{~d} z)+\int_{0}^{t} \int_{0}^{Y_{2}^{r_{2}}(s-)} \int_{\mathbb{M}_{-1} \backslash A_{r}} z_{2} \tilde{N}_{2}(\mathrm{~d} s, \mathrm{~d} u, \mathrm{~d} z) .
\end{aligned}
$$


Then $\left\{\left(Y_{1}^{r_{1}}(t), Y_{2}^{r_{2}}(t)\right): t \geq 0\right\}$ is a MSB-process with branching mechanism $\left(\Phi_{1}^{r}, \Phi_{2}^{r}\right)$. It is easy to see that $\left(Y_{1}^{r_{1}}(s), Y_{2}^{r_{2}}(s)\right)=\left(Y_{1}(s), Y_{1}(s)\right)$ for $0 \leq s<\tau_{r}$ and

$$
\begin{aligned}
& \left\{\tau_{r}>t\right\} \\
& =\left\{\max _{0<s \leq t} \Delta Y_{1}(s) \leq r_{1}, \max _{0<s \leq t} \Delta Y_{2}(s) \leq r_{2}\right\} \\
& =\left\{\int_{0}^{t} \int_{0}^{Y_{1}(s-)} \int_{A_{r}} N_{1}(\mathrm{~d} s, \mathrm{~d} u, \mathrm{~d} z)=0, \int_{0}^{t} \int_{0}^{Y_{2}(s-)} \int_{A_{r}} N_{2}(\mathrm{~d} s, \mathrm{~d} u, \mathrm{~d} z)=0\right\} \\
& =\left\{\int_{0}^{t} \int_{0}^{Y_{1}^{r_{1}}(s-)} \int_{A_{r}} N_{1}(\mathrm{~d} s, \mathrm{~d} u, \mathrm{~d} z)=0, \int_{0}^{t} \int_{0}^{Y_{2}^{r_{2}}(s-)} \int_{A_{r}} N_{2}(\mathrm{~d} s, \mathrm{~d} u, \mathrm{~d} z)=0\right\} .
\end{aligned}
$$

Note that $N_{1}$ and $N_{2}$ restricted to $(0, \infty)^{2} \times A_{r}$ are independent of $\left\{\left(Y_{1}^{r_{1}}(t), Y_{2}^{r_{2}}(t)\right): t \geq 0\right\}$. It follows that

$$
\mathbf{P}_{y}\left\{\tau_{r}>t\right\}=\mathbf{P}_{y} \exp \left\{-n_{1}\left(A_{r}\right) \int_{0}^{t} Y_{1}^{r_{1}}(s) \mathrm{d} s-n_{2}\left(A_{r}\right) \int_{0}^{t} Y_{2}^{r_{2}}(s) \mathrm{d} s\right\}
$$

Finally the desired result follows from (5.1)-(5.4).

Corollary 5.2 Suppose that both $n_{1}$ and $n_{2}$ have unbounded supports. As $r \rightarrow \infty$, we have

$$
\mathbf{P}_{y}\left\{\tau_{r} \leq t\right\} \sim\left(y_{1}, y_{2}\right) \int_{0}^{t} \mathrm{e}^{(t-s) \mathrm{H}} \mathrm{d} s\left(\begin{array}{c}
n_{1}\left(A_{r}\right) \\
n_{2}\left(A_{r}\right)
\end{array}\right)
$$

where

$$
\mathrm{H}=\left(\begin{array}{cc}
-a_{11} & \int_{\mathbb{M}} z_{2} n_{1}(\mathrm{~d} z) \\
a_{21}+\int_{\mathbb{M}_{-1}} z_{1} n_{2}(\mathrm{~d} z) & \int_{\mathbb{M}_{-1}} z_{2} n_{2}(\mathrm{~d} z)
\end{array}\right)
$$

and $\mathrm{e}^{(t-s) \mathrm{H}}=\sum_{k=0}^{\infty} \frac{(t-s)^{k} \mathrm{H}^{k}}{k !}$

Proof. For $r, q \in[0, \infty)^{2}, q_{i} \geq r_{i}, i=1,2$, we have obviously $\Phi_{i} \geq \Phi_{i}^{r} \geq \Phi_{i}^{q}, i=1,2$. Then by Proposition 5.2 we see that $\bar{V}_{i} \geq \bar{V}_{i}^{r} \geq \bar{V}_{i}^{q}, i=1,2$. It follows that

$$
1-\exp \left\{-\left\langle y, \bar{V}^{q}\left(t, n\left(A_{r}\right)\right)\right\rangle\right\} \leq \mathbf{P}_{y}\left\{\tau_{r} \leq t\right\} \leq 1-\exp \left\{-\left\langle y, \bar{V}\left(t, n\left(A_{r}\right)\right)\right\rangle\right\} .
$$

Note that $\bar{V}^{q}(t, 0+)=\bar{V}^{r}(t, 0+)=\bar{V}(t, 0+)=0$. Moreover, we can calculate that

$$
\begin{aligned}
& \frac{\partial}{\partial t} \frac{\partial}{\partial \lambda} \bar{V}_{1}(t, 0)=\mathrm{e}^{(1)}-a_{11} \frac{\partial}{\partial \lambda} \bar{V}_{1}(t, 0)+\int_{\mathbb{M}} z_{2} n_{1}(\mathrm{~d} z) \cdot \frac{\partial}{\partial \lambda} \bar{V}_{2}(t, 0) \\
& \frac{\partial}{\partial t} \frac{\partial}{\partial \lambda} \bar{V}_{2}(t, 0)=\mathrm{e}^{(2)}+\left(a_{21}+\int_{\mathbb{M}_{-1}} z_{1} n_{2}(\mathrm{~d} z)\right) \frac{\partial}{\partial \lambda} \bar{V}_{1}(t, 0)+\int_{\mathbb{M}_{-1}} z_{2} n_{2}(\mathrm{~d} z) \cdot \frac{\partial}{\partial \lambda} \bar{V}_{2}(t, 0), \\
& \frac{\partial}{\partial \lambda} \bar{V}_{1}(0,0)=\frac{\partial}{\partial \lambda} \bar{V}_{2}(0,0)=0 .
\end{aligned}
$$


We can solve the above equations to get

$$
\left(\begin{array}{c}
\frac{\partial}{\partial \lambda} \bar{V}_{1}(t, 0) \\
\frac{\partial}{\partial \lambda} \bar{V}_{2}(t, 0)
\end{array}\right)=\int_{0}^{t} \mathrm{e}^{(t-s) \mathrm{H}} \mathrm{d} s
$$

Similarly we have

$$
\left(\begin{array}{c}
\frac{\partial}{\partial \lambda} \bar{V}_{1}^{q}(t, 0) \\
\frac{\partial}{\partial \lambda} \bar{V}_{2}^{q}(t, 0)
\end{array}\right)=\int_{0}^{t} \mathrm{e}^{(t-s) \mathrm{H}_{q}} \mathrm{~d} s
$$

where

$$
\mathrm{H}_{q}=\left(\begin{array}{cc}
-a_{11}^{q} & \int_{\mathbb{M} \backslash A_{q}} z_{2} n_{1}(\mathrm{~d} z) \\
a_{21}+\int_{\mathbb{M}_{-1} \backslash A_{q}} z_{1} n_{2}(\mathrm{~d} z) & \int_{\mathbb{M}_{-1} \backslash A_{q}} z_{2} n_{2}(\mathrm{~d} z)
\end{array}\right) .
$$

By (5.5) and (5.6), as $r \rightarrow \infty$,

$$
\begin{aligned}
1-\exp \left\{-\left\langle y, \bar{V}\left(t, n\left(A_{r}\right)\right)\right\rangle\right\} & \sim\left\langle y, \bar{V}\left(t, n\left(A_{r}\right)\right)\right\rangle \\
& \sim\left(y_{1}, y_{2}\right) \int_{0}^{t} \mathrm{e}^{(t-s) \mathrm{H}} \mathrm{d} s\left(\begin{array}{c}
n_{1}\left(A_{r}\right) \\
n_{2}\left(A_{r}\right)
\end{array}\right)
\end{aligned}
$$

and

$$
\begin{aligned}
1-\exp \left\{-\left\langle y, \bar{V}^{q}\left(t, n\left(A_{r}\right)\right)\right\rangle\right\} & \sim\left\langle y, \bar{V}^{q}\left(t, n\left(A_{r}\right)\right)\right\rangle \\
& \sim\left(y_{1}, y_{2}\right) \int_{0}^{t} \mathrm{e}^{(t-s) \mathrm{H}_{q}} \mathrm{~d} s\left(\begin{array}{c}
n_{1}\left(A_{r}\right) \\
n_{2}\left(A_{r}\right)
\end{array}\right) .
\end{aligned}
$$

Then we complete the proof by noticing $\lim _{q \rightarrow \infty} \mathrm{H}_{q}=\mathrm{H}$.

\section{Exponential ergodicity in Wasserstein distances}

In order to present our results in this section, we first introduce some notations. Given two probability measures $\mu$ and $\nu$ on $\mathbb{M}$, the standard $L^{p}$-Wasserstein distance $W_{p}$ for all $p \geq 1$ is given by

$$
W_{p}(\mu, \nu)=\inf _{\Pi \in \mathcal{C}(\mu, \nu)}\left(\int_{\mathbb{M} \times \mathbb{M}}|x-y|^{p} \Pi(\mathrm{d} x, \mathrm{~d} y)\right)^{1 / p},
$$

where $|\cdot|$ denotes the Euclidean norm and $\mathcal{C}(\mu, \nu)$ stands for the set of all coupling measures of $\mu$ and $\nu$, i.e. $\mathcal{C}(\mu, \nu)$ is the collection of measures on $\mathbb{M} \times \mathbb{M}$ having $\mu$ and $\nu$ as marginals. Denote $\mathcal{P}_{p}(\mathbb{M})$ as the set of probability measures having finite moment of order $p$, it is known that $\left(\mathcal{P}_{p}(\mathbb{M}), W_{p}\right)$ becomes a Polish space.

The next theorem gives upper and lower bounds for the variations in the $L^{1}$-Wasserstein distance $W_{1}$ of the transition probabilities of the MSB-process started from two different initial states. 
Theorem 6.1 Let $\left(P_{t}\right)_{t \geq 0}$ be the transition semigroup defined by (3.10). Then for all $x, y \in \mathbb{M}$ and $t \geq 0$ we have

$$
|\langle x-y, \pi(t, 1)\rangle| \leq W_{1}\left(\delta_{x} P_{t}, \delta_{y} P_{t}\right) \leq \sum_{i=1}^{2}\left|x_{i}-y_{i}\right| \pi_{i}(t, 1),
$$

where $\delta_{x} P_{t}(\cdot):=P_{t}(x, \cdot)$ and $\pi(t, 1)$ is defined as in Proposition 3.4 with $\lambda=(1,1)$.

Proof. The proof is based on the same idea as that of Theorem 2.2 in [24. By Proposition 3.4. we see that $\int_{\mathbb{M}}\left(y_{1}+y_{2}\right) P_{t}(x, \mathrm{~d} y)=\langle x, \pi(t, 1)\rangle$. It follows from Theorem 5.10 in [4] that

$$
W_{1}\left(\delta_{x} P_{t}, \delta_{y} P_{t}\right) \geq \int_{\mathbb{M}}\left(z_{1}+z_{2}\right)\left(P_{t}(x, \mathrm{~d} z)-P_{t}(y, \mathrm{~d} z)\right)=\langle x-y, \pi(t, 1)\rangle .
$$

Similarly, $W_{1}\left(\delta_{x} P_{t}, \delta_{y} P_{t}\right) \geq\langle y-x, \pi(t, 1)\rangle$. Then the first inequality follows. On the other hand, for $x, y \in \mathbb{M}$, let $(x-y)_{ \pm}:=\left(\left(x_{1}-y_{1}\right)_{ \pm},\left(x_{2}-y_{2}\right)_{ \pm}\right)$, and $x \wedge y:=x-(x-y)_{+}=y-(x-y)_{-}$. Let $P_{t}\left(x, y, \mathrm{~d} \eta_{1}, \mathrm{~d} \eta_{2}\right)$ be the image of the product measure

$$
P_{t}\left(x \wedge y, \mathrm{~d} \gamma_{0}\right) P_{t}\left((x-y)_{+}, \mathrm{d} \gamma_{1}\right) P_{t}\left((x-y)_{-}, \mathrm{d} \gamma_{2}\right)
$$

under the mapping $\left(\gamma_{0}, \gamma_{1}, \gamma_{2}\right) \mapsto\left(\eta_{1}, \eta_{2}\right):=\left(\gamma_{0}+\gamma_{1}, \gamma_{0}+\gamma_{2}\right)$. It's not hard to see that $P_{t}\left(x, y, \mathrm{~d} \eta_{1}, \mathrm{~d} \eta_{2}\right)$ is a coupling of $P_{t}\left(x, \mathrm{~d} \eta_{1}\right)$ and $P_{t}\left(y, \mathrm{~d} \eta_{2}\right)$. Then

$$
\begin{aligned}
W_{1}\left(\delta_{x} P_{t}, \delta_{y} P_{t}\right) & \leq \int_{\mathbb{M}^{2}}\left|\eta_{1}-\eta_{2}\right| P_{t}\left(x, y, \mathrm{~d} \eta_{1}, \mathrm{~d} \eta_{2}\right) \\
& \leq \int_{\mathbb{M}} P_{t}\left((x-y)_{+}, \mathrm{d} \gamma_{1}\right) \int_{\mathbb{M}}\left(\gamma_{11}+\gamma_{12}+\gamma_{21}+\gamma_{22}\right) P_{t}\left((x-y)_{-}, \mathrm{d} \gamma_{2}\right) \\
& =\int_{\mathbb{M}}\left(\zeta_{1}+\zeta_{2}\right) P_{t}\left(\left(\left|x_{1}-y_{1}\right|,\left|x_{2}-y_{2}\right|\right), \mathrm{d} \zeta\right) \\
& =\sum_{i=1}^{2}\left|x_{i}-y_{i}\right| \pi_{i}(t, 1),
\end{aligned}
$$

where we have used the branching property $P_{t}(a, \cdot) * P_{t}(b, \cdot)=P_{t}(a+b, \cdot)$ for all $a, b \in \mathbb{M}, t \geq 0$ in the third row. Therefore the proof is finished.

Based on Theorem 6.1, we can establish the exponential ergodicity with respect to $W_{1}$. Recalling that a $2 \times 2$ matrix $H=\left[H_{i j}\right]_{2 \times 2}$ in Corollary 5.2 is defined as follows:

$$
\mathrm{H}=\left(\begin{array}{cc}
-a_{11} & \int_{\mathbb{M}} z_{2} n_{1}(\mathrm{~d} z) \\
a_{21}+\int_{\mathbb{M}_{-1}} z_{1} n_{2}(\mathrm{~d} z) & \int_{\mathbb{M}_{-1}} z_{2} n_{2}(\mathrm{~d} z)
\end{array}\right),
$$

we have the following result:

Theorem 6.2 Assume that $H_{11} H_{22}-H_{12} H_{21}>0$ and $H_{11}+H_{22}<0$. Then there exist $\lambda, \vartheta>0$ such that for any $t \geq 0$ and $x, y \in \mathbb{M}$,

$$
W_{1}\left(\delta_{x} P_{t}, \delta_{y} P_{t}\right) \leq \vartheta|x-y| \mathrm{e}^{-\lambda t} .
$$


Proof. By assumption, it is easy to see that

$$
\lambda^{2}-\left(H_{11}+H_{22}\right) \lambda+H_{11} H_{22}-H_{12} H_{21}=0
$$

has two different roots: $\lambda_{1}=2^{-1}\left(H_{11}+H_{22}+\sqrt{\Delta}\right), \lambda_{2}=\lambda_{1}-\sqrt{\Delta}$ and $\lambda_{2}<\lambda_{1}<0$, where $\Delta=\left(H_{11}-H_{22}\right)^{2}+4 H_{12} H_{21}>0$. If $H_{12}=H_{21}=0$, then $\lambda_{1}=H_{11}$ and $\lambda_{2}=H_{22}$. By Proposition 3.4, $\pi_{i}(t, 1)=\mathrm{e}^{\lambda_{i} t}$ for $i=1,2$, and the desired result follows. Next we only consider $H_{12}>0$. We can calculate that

$$
\begin{aligned}
\pi_{1}(t, 1) & =\frac{H_{11}+H_{12}-\lambda_{2}}{\sqrt{\Delta}} \mathrm{e}^{\lambda_{1} t}+\frac{\lambda_{1}-H_{11}-H_{12}}{\sqrt{\Delta}} \mathrm{e}^{\lambda_{2} t} \\
& :=\theta_{11} \mathrm{e}^{\lambda_{1} t}+\theta_{12} \mathrm{e}^{\lambda_{2} t} \\
\pi_{2}(t, 1) & =\frac{\left(H_{11}+H_{12}-\lambda_{2}\right)\left(\lambda_{1}-H_{11}\right)}{\sqrt{\Delta} H_{12}} \mathrm{e}^{\lambda_{1} t}+\frac{\left(\lambda_{1}-H_{11}-H_{12}\right)\left(\lambda_{2}-H_{11}\right)}{\sqrt{\Delta} H_{12}} \mathrm{e}^{\lambda_{2} t} \\
& :=\theta_{21} \mathrm{e}^{\lambda_{1} t}+\theta_{22} \mathrm{e}^{\lambda_{2} t} .
\end{aligned}
$$

It is easy to see that $\theta_{11}, \theta_{21}>0$. It follows from Theorem 6.1 that

$$
\begin{aligned}
W_{1}\left(\delta_{x} P_{t}, \delta_{y} P_{t}\right) & \leq\left|x_{1}-y_{1}\right|\left(\theta_{11} \mathrm{e}^{\lambda_{1} t}+\theta_{12} \mathrm{e}^{\lambda_{2} t}\right)+\left|x_{2}-y_{2}\right|\left(\theta_{21} \mathrm{e}^{\lambda_{1} t}+\theta_{22} \mathrm{e}^{\lambda_{2} t}\right) \\
& \leq|x-y|\left(\left|\theta_{11}\right|+\left|\theta_{21}\right|\right) \mathrm{e}^{\lambda_{1} t}+|x-y|\left(\left|\theta_{12}\right|+\left|\theta_{22}\right|\right) \mathrm{e}^{\lambda_{2} t} \\
& \leq\left(\theta_{11}+\theta_{21}+\left|\theta_{12}\right|+\left|\theta_{22}\right|\right)|x-y| \mathrm{e}^{\lambda_{1} t}
\end{aligned}
$$

we obtain the desired result by setting $\vartheta=\theta_{11}+\theta_{21}+\left|\theta_{12}\right|+\left|\theta_{22}\right|>0$ and $\lambda=-\lambda_{1}>0$.

Corollary 6.1 Assume the conditions of Theorem 6.2 hold. Then there exist a unique $\pi \in$ $\mathcal{P}_{1}(\mathbb{M})$ and $\vartheta, \lambda>0$ such that for any $x \in \mathbb{M}$ and $t \geq 0$

$$
W_{1}\left(\delta_{x} P_{t}, \pi\right) \leq \vartheta W_{1}\left(\delta_{x}, \pi\right) \mathrm{e}^{-\lambda t} .
$$

Proof. By Theorem 7.3 below, there exists a unique invariant measure. Arguing similarly to the proof of Theorem 3.2 in [12], one can see that $\pi \in \mathcal{P}_{1}(\mathbb{M})$, and the desired assertion is easily obtained by Theorem 6.2

\section{$7 \quad$ MSBI-processes}

Suppose that $\Phi_{1}, \Phi_{2}$ are two functions on $[0, \infty)^{2}$ defined as in (3.3)-(3.4), and there exists function $\Psi$ on $[0, \infty)^{2}$ defined by:

$$
\Psi\left(\lambda_{1}, \lambda_{2}\right)=b \lambda_{1}+\int_{\mathbb{M}}\left(1-\mathrm{e}^{-\langle\lambda, z\rangle}\right) m(\mathrm{~d} z), \quad \lambda \in \mathbb{R}_{+}^{2},
$$

where $b>0$ and $m$ is a $\sigma$-finite measure on $\mathbb{M}$ supported by $\mathbb{M} \backslash\{\mathbf{0}\}$ such that

$$
\int_{\mathbb{M}}\left(1 \wedge z_{1}+1 \wedge z_{2}\right) m(\mathrm{~d} z)<\infty
$$


A Markov process $\left\{Z(t)=\left(Z_{1}(t), Z_{2}(t)\right): t \geq 0\right\}$ is called a MSBI-process on $\mathbb{M}$, if it has transition semigroup $\left(P_{t}^{\gamma}\right)_{t \geq 0}$ uniquely determined by:

$$
\int_{\mathbb{M}} \mathrm{e}^{-\langle\lambda, y\rangle} P_{t}^{\gamma}(x, \mathrm{~d} y)=\exp \left\{-\langle x, V(t, \lambda)\rangle-\int_{0}^{t} \Psi(V(s, \lambda)) \mathrm{d} s\right\}, \quad x \in \mathbb{M}, \lambda \in \mathbb{R}_{+}^{2},
$$

where $V(t, \lambda)=\left(V_{1}(t, \lambda), V_{2}(t, \lambda)\right)$ takes values on $\mathbb{R}_{+}^{2}$ and satisfies (3.8). One can see that the semigroup defined by (7.2) is a Feller semigroup, then the MSBI-process has a càdlàg realization. We can also establish the similar result of Theorem 6.1 for MSBI-processes, indeed, we have the following:

Theorem 7.1 Let $\left(P_{t}^{\gamma}\right)_{t \geq 0}$ be the transition semigroup defined by (7.2). Assume that $\int_{\mathbb{M}}\left(z_{1}+\right.$ $\left.z_{2}\right) m(\mathrm{~d} z)<\infty$. Then for $t \geq 0$ and $x, y \in \mathbb{M}$ we have

$$
|\langle x-y, \pi(t, 1)\rangle| \leq W_{1}\left(\delta_{x} P_{t}^{\gamma}, \delta_{y} P_{t}^{\gamma}\right) \leq \sum_{i=1}^{2}\left|x_{i}-y_{i}\right| \pi_{i}(t, 1),
$$

where $\pi(t, 1)$ is defined as in Proposition 3.4 with $\lambda=(1,1)$.

Proof. The proof is based on the same idea as that of Theorem 4.1 in [24]. One can see that

$$
\begin{aligned}
\int_{\mathbb{M}}\left(y_{1}+y_{2}\right) P_{t}^{\gamma}(x, \mathrm{~d} y)= & \langle x, \pi(t, 1)\rangle+\left(b+\int_{\mathbb{M}} z_{1} m(\mathrm{~d} z)\right) \int_{0}^{t} \pi_{1}(s, 1) \mathrm{d} s \\
& +\int_{\mathbb{M}} z_{2} m(\mathrm{~d} z) \int_{0}^{t} \pi_{2}(s, 1) \mathrm{d} s,
\end{aligned}
$$

which yields that

$$
W_{1}\left(\delta_{x} P_{t}^{\gamma}, \delta_{y} P_{t}^{\gamma}\right) \geq \int_{\mathbb{M}}\left(z_{1}+z_{2}\right)\left(P_{t}^{\gamma}(x, \mathrm{~d} z)-P_{t}^{\gamma}(y, \mathrm{~d} z)\right)=\langle x-y, \pi(t, 1)\rangle,
$$

similarly, $W_{1}\left(\delta_{x} P_{t}^{\gamma}, \delta_{y} P_{t}^{\gamma}\right) \geq\langle y-x, \pi(t, 1)\rangle$, and the first inequality follows. Next, we want to construct a coupling measure of $P_{t}^{\gamma}(x, \cdot)$ and $P_{t}^{\gamma}(y, \cdot)$. It is known that there exists a family of probability measures $\left(\gamma_{t}\right)_{t \geq 0}$ such that $P_{t}^{\gamma}(x, \cdot)=P_{t}(x, \cdot) * \gamma_{t}(\cdot)$ for $t \geq 0, x \in \mathbb{M}$, and

$$
\int_{\mathbb{M}} \mathrm{e}^{-\langle\lambda, y\rangle} \gamma_{t}(\mathrm{~d} y)=\exp \left\{-\int_{0}^{t} \Psi(V(s, \lambda)) \mathrm{d} s\right\},
$$

we call $\left(\gamma_{t}\right)_{t \geq 0}$ a skew convolution semigroup associated with $\left(P_{t}\right)_{t \geq 0}$; see, e.g., Chapter 9 in [21. Let $P_{t}\left(x, y, \mathrm{~d} \eta_{1}, \mathrm{~d} \eta_{2}\right)$ be the coupling measure of $P_{t}\left(x, \mathrm{~d} \eta_{1}\right)$ and $P_{t}\left(y, \mathrm{~d} \eta_{2}\right)$ constructed in the proof of Theorem 6.1 and $P_{t}^{\gamma}\left(x, y, \mathrm{~d} \sigma_{1}, \mathrm{~d} \sigma_{2}\right)$ be the image of $\gamma_{t}\left(\mathrm{~d} \eta_{0}\right) P_{t}\left(x, y, \mathrm{~d} \eta_{1}, \mathrm{~d} \eta_{2}\right)$ under the mapping $\left(\eta_{0}, \eta_{1}, \eta_{2}\right) \mapsto\left(\sigma_{1}, \sigma_{2}\right)=\left(\eta_{0}+\eta_{1}, \eta_{0}+\eta_{2}\right)$. By the relation $P_{t}^{\gamma}(x, \cdot)=P_{t}(x, \cdot) * \gamma_{t}(\cdot)$ we see that $P_{t}^{\gamma}\left(x, y, \mathrm{~d} \sigma_{1}, \mathrm{~d} \sigma_{2}\right)$ is a coupling measure of $P_{t}^{\gamma}\left(x, \mathrm{~d} \sigma_{1}\right)$ and $P_{t}^{\gamma}\left(y, \mathrm{~d} \sigma_{2}\right)$. It follows that

$$
\begin{aligned}
W_{1}\left(\delta_{x} P_{t}^{\gamma}, \delta_{y} P_{t}^{\gamma}\right) & \leq \int_{\mathbb{M}^{2}}\left|\sigma_{1}-\sigma_{2}\right| P_{t}^{\gamma}\left(x, y, \mathrm{~d} \sigma_{1}, \mathrm{~d} \sigma_{2}\right) \\
& =\int_{\mathbb{M}} \gamma_{t}\left(\mathrm{~d} \eta_{0}\right) \int_{\mathbb{M}^{2}}\left|\eta_{1}-\eta_{2}\right| P_{t}\left(x, y, \mathrm{~d} \eta_{1}, \mathrm{~d} \eta_{2}\right) \\
& =\int_{\mathbb{M}^{2}}\left|\eta_{1}-\eta_{2}\right| P_{t}\left(x, y, \mathrm{~d} \eta_{1}, \mathrm{~d} \eta_{2}\right) \leq \sum_{i=1}^{2}\left|x_{i}-y_{i}\right| \pi_{i}(t, 1),
\end{aligned}
$$


where the last inequality follows from Theorem 6.1,

By a similar argument of Theorem 6.2, we have the following:

Theorem 7.2 Assume that $H_{11} H_{22}-H_{12} H_{21}>0$ and $H_{11}+H_{22}<0$. Then there exist $\lambda, \vartheta>0$ such that for any $t \geq 0$ and $x, y \in \mathbb{M}$,

$$
W_{1}\left(\delta_{x} P_{t}^{\gamma}, \delta_{y} P_{t}^{\gamma}\right) \leq \vartheta|x-y| \mathrm{e}^{-\lambda t}
$$

\subsection{The construction of MSBI-processes by stochastic equations}

We now give a construction of MSBI-processes by stochastic equations. Let us consider the following stochastic equation system:

$$
\begin{aligned}
Z_{1}(t)=Z_{1}(0) & +\int_{0}^{t}\left(b-a_{11} Z_{1}(s)+a_{21} Z_{2}(s)\right) \mathrm{d} s+\int_{0}^{t} \sqrt{2 \alpha Z_{1}(s)} \mathrm{d} B(s) \\
& +\int_{0}^{t} \int_{0}^{Z_{1}(s-)} \int_{\mathbb{M}} z_{1} \tilde{N}_{1}(\mathrm{~d} s, \mathrm{~d} u, \mathrm{~d} z)+\int_{0}^{t} \int_{\mathbb{M}} z_{1} M(\mathrm{~d} s, \mathrm{~d} z) \\
& +\int_{0}^{t} \int_{0}^{Z_{2}(s-)} \int_{\mathbb{M}_{-1}} z_{1} N_{2}(\mathrm{~d} s, \mathrm{~d} u, \mathrm{~d} z), \\
Z_{2}(t)=Z_{2}(0) & +\int_{0}^{t} \int_{0}^{Z_{1}(s-)} \int_{\mathbb{M}_{2}} z_{2} N_{1}(\mathrm{~d} s, \mathrm{~d} u, \mathrm{~d} z)+\int_{0}^{t} \int_{\mathbb{M}} z_{2} M(\mathrm{~d} s, \mathrm{~d} z) \\
& +\int_{0}^{t} \int_{0}^{Z_{2}(s-)} \int_{\mathbb{M}_{-1}} z_{2} N_{2}(\mathrm{~d} s, \mathrm{~d} u, \mathrm{~d} z)
\end{aligned}
$$

where $b \geq 0, M(\mathrm{~d} s, \mathrm{~d} z)$ is a Poisson random measure on $[0, \infty) \times \mathbb{M}$ with intensity measure $\mathrm{d} \operatorname{sm}(\mathrm{d} z)$, other coefficients are the same in section 4. Furthermore, we assume that those random elements are independent of each other. By a modification of the proof of section 4 as well as in [26], we see that (17.3) -(7.4) has a unique strong solution and it is a MSBI-process with branching mechanism $\left(\Phi_{1}, \Phi_{2}\right)$ defined by (3.3)-(3.4) and immigration mechanism $\Psi$ defined by (7.1).

\subsection{Stationary distribution}

In order to characterize the stationary distribution of MSBI-processes, we need to estimate the upper bound and lower bound of $|V(t, \lambda)|$ for $t>0, \lambda \in \mathbb{R}_{+}^{2}$, which will play an important role in the sequel.

Lemma 7.1 Let $\left(Y_{t}\right)_{t \geq 0}$ be a MSB-process with semigroup $\left(P_{t}\right)_{t \geq 0}$ satisfying (3.10). Let $H=$ $\left[H_{i j}\right]_{2 \times 2}$ be a $2 \times 2$ matrix defined as in Corollary [5.2. Suppose that all the eigenvalues of $H$ have strictly negative real parts. Then there exist some strictly positive constants $c_{1}(\lambda)$ and $c_{2}$, where $c_{1}$ depends on $\lambda$ such that

$$
|V(t, \lambda)| \leq c_{1}(\lambda) \exp \left\{-c_{2} t\right\}, \quad \lambda \in \mathbb{R}_{+}^{2}, \quad t \geq 0 .
$$


Proof. We follow the same calculations in Proposition 3.4 to see that

$$
\left(\begin{array}{c}
\frac{\partial V_{1}(t, 0+)}{\partial \lambda_{1}} \\
\frac{\partial V_{2}(t, 0+)}{\partial \lambda_{1}}
\end{array}\right)=\mathrm{e}^{t H}\left(\begin{array}{l}
1 \\
0
\end{array}\right)
$$

and so

$$
\int_{\mathbb{M}} y_{1} P_{t}(x, \mathrm{~d} y)=\left(\begin{array}{l}
x_{1} \\
x_{2}
\end{array}\right)^{\mathrm{T}} \mathrm{e}^{t H}\left(\begin{array}{l}
1 \\
0
\end{array}\right)
$$

Similarly,

$$
\int_{\mathbb{M}} y_{2} P_{t}(x, \mathrm{~d} y)=\left(\begin{array}{l}
x_{1} \\
x_{2}
\end{array}\right)^{\mathrm{T}} \mathrm{e}^{t H}\left(\begin{array}{l}
0 \\
\\
1
\end{array}\right)
$$

By Jensen's inequality we deduce that for all $x=\left(x_{1}, x_{2}\right) \in \mathbb{M}$

$$
\left(\begin{array}{l}
x_{1} \\
x_{2}
\end{array}\right)^{\mathrm{T}}\left(\begin{array}{c}
V_{1}(t, \lambda) \\
V_{2}(t, \lambda)
\end{array}\right) \leq\left(\begin{array}{l}
x_{1} \\
x_{2}
\end{array}\right)^{\mathrm{T}} \mathrm{e}^{t H}\left(\begin{array}{c}
\lambda_{1} \\
\lambda_{2}
\end{array}\right) .
$$

Since all the eigenvalues of $H$ have strictly negative real parts, there exist some strictly positive $c, c_{2}>0$ such that for all $t>0$

$$
\left\|\mathrm{e}^{t H}\right\|:=\sup _{|x|=1}\left|\mathrm{e}^{t H} x\right| \leq c \mathrm{e}^{-c_{2} t}
$$

see,e.g., equation (2.8) in [30], which implies that $|V(t, \lambda)| \leq|\lambda| c \mathrm{e}^{-c_{2} t}$, we finish the proof by setting $c_{1}(\lambda)=|\lambda| c$.

Lemma 7.2 Under the conditions of Lemma 7.1, for every $\lambda \in \mathbb{R}_{+}^{2}$, there exist two strictly positive constants $A(\lambda)$ and $B(\lambda)$ such that

$$
V_{1}(t, \lambda) \geq \lambda_{1} \mathrm{e}^{-A(\lambda) t}, \quad V_{2}(t, \lambda) \geq \lambda_{2} \mathrm{e}^{-B(\lambda) t}, \quad t \geq 0 .
$$

Proof.

$$
\begin{gathered}
V_{1}(t, \lambda)=\lambda_{1}+\int_{0}^{t}\left\{H_{11} V_{1}(s, \lambda)+H_{12} V_{2}(s, \lambda)-\alpha V_{1}^{2}(s, \lambda)\right. \\
\left.-\int_{\mathbb{M}}\left(\mathrm{e}^{-\langle V(s, \lambda), z\rangle}-1+\langle V(s, \lambda), z\rangle\right) n_{1}(\mathrm{~d} z)\right\} \mathrm{d} s, \\
V_{2}(t, \lambda)=\lambda_{2}+\int_{0}^{t}\left\{a_{21} V_{1}(s, \lambda)+\int_{\mathbb{M}_{-1}}\left(1-\mathrm{e}^{-\langle V(s, \lambda), z\rangle}\right) n_{2}(\mathrm{~d} z)\right\} \mathrm{d} s .
\end{gathered}
$$

It follows from Lemma 7.1, the comparison theorem and the fact

$$
\mathrm{e}^{-\lambda x}-1+\lambda x \leq\left(\frac{\lambda^{2}}{2}+\lambda\right)\left(x \wedge x^{2}\right), \quad x, \lambda \geq 0
$$


that there exists $A(\lambda)=\left|H_{11}-\kappa-(\alpha+\kappa / 2) c_{1}(\lambda)\right|$, where $\kappa=\int_{\mathbb{M}} z_{1} \wedge z_{1}^{2} n_{1}(\mathrm{~d} z)$ such that

$$
V_{1}(t, \lambda) \geq \lambda_{1} \mathrm{e}^{-A(\lambda) t}, \quad t \geq 0, \quad \lambda \in \mathbb{R}_{+}^{2} .
$$

On the other hand, let $\theta=n_{2}\left(\mathbb{R}_{+} \times\{-1\}\right)<\infty$,

$$
\begin{aligned}
V_{2}(t, \lambda) & \geq \lambda_{2}-\theta \int_{0}^{t}\left(\mathrm{e}^{V_{2}(s, \lambda)}-1\right) \mathrm{d} s \\
& \geq \lambda_{2}-2 \theta \int_{0}^{t}\left(\mathrm{e}^{c_{1}(\lambda) \mathrm{e}^{-c_{2} s}} V_{2}(s, \lambda)\right) \mathrm{d} s \\
& \geq \lambda_{2}-2 \theta \mathrm{e}^{c_{1}(\lambda)} \int_{0}^{t} V_{2}(s, \lambda) \mathrm{d} s
\end{aligned}
$$

by the comparison theorem we deduce that $V_{2}(t, \lambda) \geq \lambda_{2} \mathrm{e}^{-2 \theta \mathrm{e}^{c_{1}(\lambda)} t}$, and we obtain the desired result by setting $B(\lambda)=2 \theta \mathrm{e}^{c_{1}(\lambda)}$.

We now give our main result.

Theorem 7.3 Let $\left(Z_{t}\right)_{t \geq 0}$ be a MSBI-process with semigroup $\left(P_{t}^{\gamma}\right)_{t \geq 0}$ satisfying (7.2). Suppose that all the eigenvalues of $H$ have strictly negative real parts. Then $P_{t}^{\gamma}(x, \cdot)$ converges to a probability measure $\pi$ on $\mathbb{M}$ as $t \rightarrow \infty$ for all $x \in \mathbb{M}$ if and only if

$$
\int_{\{|z| \geq 1\}} \log |z| m(\mathrm{~d} z)<\infty
$$

Proof. By Lemma 7.1 we have $|V(t, \lambda)| \rightarrow 0$ as $t \rightarrow \infty$. Suppose that $\left(Z_{t}\right)_{t \geq 0}$ has a stationary distribution $\pi$, one can see that

$$
\int_{\mathbb{M}} \mathrm{e}^{-\langle\lambda, y\rangle} \pi(\mathrm{d} y)=\exp \left\{-\int_{0}^{\infty} \Psi(V(s, \lambda)) \mathrm{d} s\right\}, \quad \lambda \in \mathbb{R}_{+}^{2},
$$

which implies that $\int_{0}^{\infty} \Psi(V(s, \lambda)) \mathrm{d} s<\infty$ for all $\lambda \in \mathbb{R}_{+}^{2}$. And so

$$
\int_{0}^{\infty} \mathrm{d} s \int_{\{|z| \geq 1\}}\left(1-\mathrm{e}^{-\left(\lambda_{1} \wedge \lambda_{2}\right) \exp \{-[A(\lambda)+B(\lambda)] s\}\left(z_{1}+z_{2}\right)}\right) m(\mathrm{~d} z)<\infty .
$$

If we set $C(\lambda):=\left|H_{11}\right|+\vartheta+(\alpha+\vartheta / 2) c_{1}(\lambda)+2 \theta \mathrm{e}^{c_{1}(\lambda)}>0$ for all $\lambda \in \mathbb{R}_{+}^{2}$, then $C(\lambda) \geq A(\lambda)+B(\lambda)$. Choose a proper $\tilde{\lambda}$ such that $\tilde{\lambda}_{1} \wedge \tilde{\lambda}_{2}>0$ and let $t:=\mathrm{e}^{-C(\tilde{\lambda}) s}|z|$, we have $\mathrm{d} t=-C(\tilde{\lambda}) t \mathrm{~d} s$, and

$$
\int_{\{|z| \geq 1\}} m(\mathrm{~d} z) \int_{0}^{|z|} \frac{1-\mathrm{e}^{-\left(\tilde{\lambda}_{1} \wedge \tilde{\lambda}_{2}\right) t}}{t} \mathrm{~d} t<\infty
$$

which yields that

$$
\int_{\{|z| \geq 1\}} \log |z| m(\mathrm{~d} z)<\infty
$$

since

$$
\int_{0}^{|z|} \frac{1-\mathrm{e}^{-\left(\tilde{\lambda}_{1} \wedge \tilde{\lambda}_{2}\right) t}}{t} \mathrm{~d} t \sim \log |z| \quad \text { as }|z| \rightarrow \infty
$$


On the other hand, it suffices to prove $\int_{0}^{\infty} \int_{\mathbb{M}}\left(1-\mathrm{e}^{-\langle V(s, \lambda), z\rangle}\right) \mathrm{d} s m(\mathrm{~d} z)<\infty$ for all $\lambda \in R_{+}^{2}$ provided $\int_{\{|z| \geq 1\}} \log |z| m(\mathrm{~d} z)<\infty$. From Lemma 7.1 and Fubini's theorem

$$
\begin{aligned}
\int_{0}^{\infty} \mathrm{d} s \int_{\mathbb{M}}\left(1-\mathrm{e}^{-\langle V(s, \lambda), z\rangle}\right) m(\mathrm{~d} z) & \leq \int_{0}^{\infty} \mathrm{d} s \int_{\mathbb{M}}\left(1-\mathrm{e}^{-c_{1}(\lambda) \mathrm{e}^{-c_{2} s}\left(z_{1}+z_{2}\right)}\right) m(\mathrm{~d} z) \\
& =\int_{0}^{\infty} \mathrm{d} s \int_{\{|z|<1\}}\left(1-\mathrm{e}^{-c_{1}(\lambda) \mathrm{e}^{-c_{2} s}\left(z_{1}+z_{2}\right)}\right) m(\mathrm{~d} z) \\
& +\int_{0}^{\infty} \mathrm{d} s \int_{\{|z| \geq 1\}}\left(1-\mathrm{e}^{-c_{1}(\lambda) \mathrm{e}^{-c_{2} s}\left(z_{1}+z_{2}\right)}\right) m(\mathrm{~d} z) \\
& :=I_{*}(\lambda)+I^{*}(\lambda) .
\end{aligned}
$$

For $I_{*}(\lambda)$, by a change of variables $t:=c_{1}(\lambda) \mathrm{e}^{-c_{2} s}\left(z_{1}+z_{2}\right)$ we get

$$
\begin{aligned}
I_{*}(\lambda) & =\frac{1}{c_{2}} \int_{\{|z|<1\}} m(\mathrm{~d} z) \int_{0}^{c_{1}(\lambda)\left(z_{1}+z_{2}\right)} \frac{1-\mathrm{e}^{-t}}{t} \mathrm{~d} t \\
& \leq \frac{c_{1}(\lambda)}{c_{2}} \int_{\{|z|<1\}}\left(z_{1}+z_{2}\right) m(\mathrm{~d} z)<\infty,
\end{aligned}
$$

where the last inequality follows from $\int_{\mathbb{M}}\left(1 \wedge z_{1}+1 \wedge z_{2}\right) m(\mathrm{~d} z)<\infty$. Moreover, by a change of variables $t:=c_{1}(\lambda) \mathrm{e}^{-c_{2} s}|z|$, we have

$$
\begin{aligned}
I^{*}(\lambda) & =\frac{1}{c_{2}} \int_{\{|z| \geq 1\}} m(\mathrm{~d} z) \int_{0}^{c_{1}(\lambda)|z|} \frac{1-\mathrm{e}^{-t \frac{\left(z_{1}+z_{2}\right)}{|z|}}}{t} \mathrm{~d} t \\
& \leq \frac{1}{c_{2}} \int_{\{|z| \geq 1\}} m(\mathrm{~d} z) \int_{0}^{c_{1}(\lambda)|z|} \frac{1-\mathrm{e}^{-2 t}}{t} \mathrm{~d} t,
\end{aligned}
$$

which implies that $I^{*}(\lambda)<\infty$ by observing

$$
\int_{0}^{c_{1}(\lambda)|z|} \frac{1-\mathrm{e}^{-2 t}}{t} \mathrm{~d} t \sim \log |z|, \quad|z| \rightarrow \infty
$$

and $\int_{\{|z| \geq 1\}} \log |z| m(\mathrm{~d} z)<\infty$.

Corollary 7.1 Assume $H_{11} H_{22}-H_{12} H_{21}>0$ and $H_{11}+H_{22}<0$ hold. Moreover, suppose that Lévy measure $m$ satisfies $\int_{\{|z|>1\}}|z| m(\mathrm{~d} z)<\infty$. Then there exist $\lambda, \vartheta>0$ and unique $\pi \in \mathcal{P}_{1}(\mathbb{M})$ such that for any $t \geq 0$ and $x \in \mathbb{M}$,

$$
W_{1}\left(\delta_{x} P_{t}^{\gamma}, \pi\right) \leq \vartheta W_{1}\left(\delta_{x}, \pi\right) \mathrm{e}^{-\lambda t}
$$

Proof. It follows from Theorem 7.3 and assumptions that there exists a unique stationary distribution $\pi$. We can derive easily that $\mathbf{E}_{x}\left[\left|Z_{t}\right|\right]<\infty$ for all $t \geq 0$ and $x \in \mathbb{M}$ by the assumption $\int_{\{|z|>1\}}|z| m(\mathrm{~d} z)<\infty$. By a modification of the proof of Corollary 6.1, we have $\pi \in \mathcal{P}_{1}(\mathbb{M})$ and the desired result follows from Theorem 7.2 


\section{References}

[1] Athreya,K.B. and Ney,P.E.(1972): Branching Processes. Springer, Berlin.

[2] Bertoin,J.; Fontbona,J. and Martínez,S.(2008): On prolific indivials in a supercritical continuous-state branching process. J. Appl. Probab. 45, 714-726.

[3] Bertoin,J. and Le Gall,J.F.(2006): Stochastic flows associated to coalescent processes III: Limit theorems. Illinois J. Math. 50, 147-181.

[4] Chen,M.(2004): From Markov Chains to Non-Equilibrium Particle Systems. 2nd Ed. World Sci., River Edge, NJ.

[5] Dawson,D.A. and Li,Z.(2006): Skew convolution semigroups and affine Markov processes. Ann. Probab. 34, 1103-1142.

[6] Dawson,D.A. and Li,Z.(2012): Stochastic equations, flows and measure-valued processes. Ann. Probab. 40, 813-857.

[7] Dellacherie,C. and Meyer,P.A.(1982): Probabilites and Potential. Chapters V-VIII. NorthHolland, Amsterdam.

[8] Ethier,S.N. and Kurtz,T.G.(1986): Markov Processes: Characterization and Convergence. John Wiley and Sons Inc.,New York.

[9] Feketa,D.; Fontbona,J. and Kyprianou,A.E.(2019a): Skeletal stochastic differential equations for contin uous-state branching processes. J. Appl. Probab. 56, 1122-1150.

[10] Feketa,D.; Fontbona,J. and Kyprianou,A.E.(2019b): Skeletal stochastic differential equations for superprocesses. Available at: 1904.05966v3.

[11] Feller,W.(1951): Diffusion processes in genetics. In: Proceedings 2nd Berkeley Symp. Math. Statist. Probab., 1950, 227-246. Univ. of California Press, Berkeley and Los Angeles.

[12] Friesen,M.; Jin,P.; Kremer,J. and Rüdiger,B. (2019): Exponential ergodicity for stochastic equations of nonnegative processes with jumps. Available at:rxiv:1902.02833

[13] Fu,Z. and Li,Z.(2010): Stochastic equations of non-negative processes with jumps. Stochastic Process. Appl. 120, 306-330.

[14] He,X. and Li,Z.(2016): Distributions of jumps in a continuous-state branching process with immigration. J.Appl.Probab. 53, 1166-1177.

[15] Ikeda,N. and Watanabe,S.(1989): Stochastic Differential Equations and Diffusion Processes. North-Holland/Kodansha,Amsterdam/Tokyo.

[16] Jiřina, M.(1958): Stochastic branching processes with continuous state space. Czechoslovak Math. J. 8, 292-313.

[17] Jiao,Y.; Ma,C. and Scotti,S.(2017): Alpha-CIR model with branching processes in sovereign interest rate modeling. Finance Stoch. 21, 789-813.

[18] Jin, P.; Kremer, J. and Rüdiger, B. (2020): Existence of limiting distribution for affine processes. J. Math. Anal. Appl. 486, 123912, 31 pp. 
[19] Kawazu,K. and Watanabe,S.(1971): Branching processes with immigration and related limit theorems. Theory Probab. Appl. 16, 36-54.

[20] Li,Z.(2006): A limit theorem for discrete Galton-Watson branching processes with immigration. J. Appl. Probab. 43, 289-295.

[21] Li,Z.(2011):Measure-Valued Branching Markov Processes. Probability and its Applications. Springer, Berlin.

[22] Li,Z. and Ma,C.(2015): Asymptotic properties of estimators in a stable Cox-Ingersoll-Ross model. Stochastic Process. Appl. 125, 3196-3233.

[23] Li, Z. (2020): Continuous-state branching processes with immigration. A Chapter in: From Probability to Finance, Mathematical Lectures from Peking University, pp. 1-69. Edited by Y. Jiao. Springer, Singapore.

[24] Li, Z. (2020+): Ergodicities and exponential ergodicities of Dawson-Watanabe type processes. To appear in Theory Probab. Appl. Available at arXiv: 2002.09111.

[25] Ma,C.(2009): A limit theorem of two-type Galton-Watson branching processes with immigration.Statistics and Probability Letters. 79, 1710-1716.

[26] Ma,R.(2013): Stochastic Equations for Two-type Continuous-state Branching Processes with Immigration. Acta Math.Sinica,Engl.Ser.Mar. 29, 287-294.

[27] Ma,R. (2014): Stochastic equations for two-type continuous-state branching processes with immigration and competition. Statistics and Probability Letters. 91, 83-89.

[28] Pardoux,E. (2016): Probabilistic Models of Population Evolution: Scaling Limits, Genealogies and Interactions. Springer, Switzerland.

[29] Pinsky,M.A. (1972): Limit theorems for continuous state branching processes with immigration. Bull. Amer.Math.Soc. 78, 242-244.

[30] Sato, K. and Yamazato, M. (1984): Operator-self-decomposable distributions as limit distributions of processes of Ornstein-Uhlenbeck type. Stochastic Process. Appl. 17, 73-100. 